Supplementary Information for

\title{
Bioinspired Electro-Responsive Multispectral Controllable Dye-Doped Liquid Crystal Yolk-Shell Microcapsules for Advanced Textiles
}

Mingfei Sheng ${ }^{\text {a }}$, Liping Zhang ${ }^{a^{*}}$, Shan Jiang ${ }^{\text {a }}$, Li Yang ${ }^{\text {a }}$, Farah Zaaboul ${ }^{\text {b }}$, and Shaohai Fu ${ }^{a^{*} \dagger}$

${ }^{\text {a }}$ Key Laboratory of Science \& Technology of Eco-Textile, Jiangnan University, Ministry of Education, Wuxi, Jiangsu 214122, China

${ }^{\mathrm{b}}$ School of Food Science and Technology, Jiangnan University, Wuxi, Jiangsu 214122, China

*Corresponding Authors E-mail: zhangliping0328@163.com.(L.Z.); shaohaifu@ hotmail.com.(S.F.)

$\dagger$ Non-Corresponding Authors Information:

Mingfei Sheng: shengmingfei1028@163.com; Shan Jiang: 921976275@qq.com;

Li Yang: 2381867361@qq.com; Farah Zaaboul: farahzaaboul@hotmail.fr. 


\section{Table of Contents}

\section{Supplementary Materials \& Methods}

Table S1. The physical properties of the liquid crystal E7.

Chart S1. Composition and chemical structure of E7 and dichroic dye

\section{Supplementary Results \& Discussion}

\subsection{Preparation and Characterization of Magnetic Seeds}

Figure S1. (a) Schematic illustration of the proposed formation mechanism and synthesis process of the magnetic seed, and the photograph of $\mathrm{Fe}_{3} \mathrm{O}_{4}, \mathrm{Fe}_{3} \mathrm{O}_{4} @ \mathrm{SiO}_{2}, \mathrm{Fe}_{3} \mathrm{O}_{4} @ \mathrm{SiO}_{2} @$ VTES dispersion with and without magnetic field; The TEM images and particle size distribution (PSD) of the modified (b) $\mathrm{Fe}_{3} \mathrm{O}_{4},(\mathrm{c}) \mathrm{Fe}_{3} \mathrm{O}_{4} @ \mathrm{SiO}_{2}$, and (d) $\mathrm{Fe}_{3} \mathrm{O}_{4} @ \mathrm{SiO}_{2} @$ VTES particles.

\section{Note for Figure S1.}

Figure S2. SEM-EDS mapping images of the synthesized (a) $\mathrm{Fe}_{3} \mathrm{O}_{4}$, (b) $\mathrm{Fe}_{3} \mathrm{O}_{4} @ \mathrm{SiO}_{2}$, (c) $\mathrm{Fe}_{3} \mathrm{O}_{4} @ \mathrm{SiO}_{2} @$ VTES particles. EDS analysis of the synthesized (d) $\mathrm{Fe}_{3} \mathrm{O}_{4}$, (e) $\mathrm{Fe}_{3} \mathrm{O}_{4} @ \mathrm{SiO}_{2}$, (f) $\mathrm{Fe}_{3} \mathrm{O}_{4} @ \mathrm{SiO}_{2} @ \mathrm{VTES}$, and (g) $\mathrm{Fe}_{3} \mathrm{O}_{4} @ \mathrm{SiO}_{2} @ \mathrm{VTES} @$ DDLC@PMMA particles.

Figure S3. (a) TGA and (b) DTG analysis of the modified $\mathrm{Fe}_{3} \mathrm{O}_{4}, \mathrm{Fe}_{3} \mathrm{O}_{4} @ \mathrm{SiO}_{2}$, and $\mathrm{Fe}_{3} \mathrm{O}_{4} @ \mathrm{SiO}_{2} @ \mathrm{VTES}$ particles.

\subsection{Characterization and Performance of EM-DDLC-YS-Ms}

Figure S4. SEM-EDS mapping images of the EM-DDLC-YS-Ms.

Figure S5. OM images (reflected light mode, R-light; transmitted light mode, T-light) and POM image (R-light) ( $\times 500$ magnification) of the (a) EM-DDLC-YS-Ms emulsion and (b) dry synthesized EM-DDLC-YS-Ms particles. (scale bar, $50 \mu \mathrm{m}$ )

Figure S6. (a) PSD analysis and (b) TGA-DTG analysis of the synthesized EM-DDLC-YS-Ms particles; (c) FTIR-ATR and (d) DSC analysis of the EM-DDLC-YS-Ms; (e) HP-POM images of EM-DDLC-YS-Ms at different temperatures.

\section{Note for Figure S6.}

Figure S7. (a) FOS transmittance test and (e) K/S value of EM-DDLC-YS-Ms (for 1\#), DDLC (for 2\#), dichroic dye (for 3\#), and DDLC-Ms films (for 4\#).

Figure S8. Photographs of pure DDLC (for 1\#), $\mathrm{Fe}_{3} \mathrm{O}_{4}$ particle doped DDLC emulsion (for 2\#), carbon black doped DDLC emulsion (for 3\#), black pigment paste doped DDLC emulsion (for 4\#), and EM-DDLC-YS-Ms emulsion (for 5\#).

\section{Note for Figures S7 and S8.}

\subsection{Supernumerary Photoelectric Performance of EM-DDLC-YS-Ms}

\section{Note for Figure 3.}

\subsection{Demo of Recyclable Process of EM-DDLC-YS-Ms Film}

Figure S9. (a) The schematic of the EO property of EM-DDLC-YS-Ms measurements under POM. (b) Photographs, OM images, and viewing angle test images of EM-DDLC-YS-Ms device with applied and removed voltage. (c) Wavelength dependencies of the reflectance of EM-DDLC-YS-Ms 
device under the different applied voltage. (d) POM images of EM-DDLC-YS-Ms dynamic change by applied and removed voltage.

Figure S10. Schematic illustration and photographs of the proposed recyclable process for EM-DDLC-YS-Ms: (a) the photograph, OM image, and POM image of EM-DDLC-Ms-YS-Ms film; magnetic separation demo of the (b) EM-DDLC-YS-Ms film solution, (c) EM-DDLC-YS-Ms emulsion and (e) magnetic seeds from ground microcapsules; (d) the demonstration of electrical stimulation response process of (d) separated EM-DDLC-YS-Ms and (f) purified DDLC by ethanol.

\section{Note for Figure S10.}

\subsection{Supernumerary Magnetically Controllable Performance of EM-DDLC-YS-Ms}

Figure S11. Maximum reflectance of the magnetically controlled EM-DDLC-YS-Ms device at 650 $\mathrm{nm}$ during the applied and removed electric field cycles.

\section{Note for Figure 4 and Figure S11.}

\subsection{Characterization and Performance of EM-DDLC-YS-Ms Non-Woven-Fabric}

Figure S12. OM/POM images demonstrating the reversible change of the as-made EMs-Fabric in response to the electric field: the OM images of (a) OFF State and (b) ON State, and POM image of (c) OFF State and (d) ON State (reflected light mode, R-light) $(\times 500$ magnification).

Figure S13. (a) Reflectivity curve of the EMs-Fabric with various voltages applied. (b) Voltage dependence of the reflectance for the intelligent fabric device. (c) Maximum reflectance of the EMs-Fabric device at 550nm during the applied and removed electric field cycles. (d) The comparison of the Vis-NIR transmittance spectra of the DDLC-Ms non-woven fabric device (prepared by the same method as the EM-DDLC-YS-Ms non-woven fabric device but without the magnetic seeds) under on/off state $(0 \mathrm{~V} / 10 \mathrm{~V})$. (e) Vis-NIR reflectivity curve of the EMs-Fabric with various voltages applied. (f) Reflectance spectra of the film at the initial state and after 100 cycles. (g) Photographs, and (h) infrared thermic images of the EMs-Fabric device under applied voltages with time prolonged (recorded at a distance of $20 \mathrm{~cm}$ from the device).

\section{Note for Figure 5, Figure S12, and Figure S13.}

Figure S14. The comparison of the $S$ parameter (a) $S 11$ (b) $S 12$; (c) $S 21$; (d) $S 22$ of electromagnetic shielding sample (EM-DDLC-YS-Ms non-woven for T-sample, and the DDLC-Ms non-woven prepared by the same method as the EM-DDLC-YS-Ms non-woven device but without the magnetic seeds for C-sample).

Figure S15. The comparison of the (a) $R$ and (b) $T$ parameters of electromagnetic shielding sample (EM-DDLC-YS-Ms non-woven for T-sample, and the DDLC-Ms non-woven prepared by the same method as the EM-DDLC-YS-Ms non-woven device but without the magnetic seeds for C-sample).

Figure S16. The comparison of the $S E_{T}$ parameters of electromagnetic shielding sample (EM-DDLC-YS-Ms non-woven for T-sample, and the DDLC-Ms non-woven prepared by the same method as the EM-DDLC-YS-Ms non-woven device but without the magnetic seeds for C-sample).

\section{Note for Figure S14, Figure S15, and Figure S16.}

Figure S17. The comparison of the complex permittivity (a) $\varepsilon^{\prime}$ and (b) $\varepsilon^{\prime \prime}$, and complex permeability (c) $\mu^{\prime}$, (d) $\mu^{\prime \prime}$ of electromagnetic wave absorbing sample (EM-DDLC-YS-Ms non-woven for T-sample, and the DDLC-Ms non-woven prepared by the same method as the EM-DDLC-YS-Ms non-woven device but without the magnetic seeds for C-sample).

Figure S18. The comparison of the dielectric loss (a) $\tan \delta_{\varepsilon}$ and magnetic loss (b) $\tan \delta_{m}$ of electromagnetic wave absorbing sample (EM-DDLC-YS-Ms non-woven for T-sample, and the 
DDLC-Ms non-woven prepared by the same method as the EM-DDLC-YS-Ms non-woven device but without the magnetic seeds for C-sample).

Figure S19. The comparison of the reflection loss curves of electromagnetic wave absorbing samples with different thickness: (a) EM-DDLC-YS-Ms non-woven for T-sample, (b, c) the DDLC-Ms non-woven prepared by the same method as the EM-DDLC-YS-Ms non-woven device but without the magnetic seeds for C-sample.

Note for Figure S17, Figure S18, and Figure S19.

Figure S20. Photographs of EMs-Fabric prepared by other different DDLC and CLC systems under applied (I, II, III, IV) and removed (V, VI, VII, VIII) electric field state.

\section{Supplementary Movies}

Movie S1. The color change of the EM-DDLC-YS-Ms during applying and removing voltage.

\section{Supplementary Description}

\section{Supplementary References}




\section{Supplementary Materials \& Methods}

1.1. Mean Particle Size and Particle Size Distribution (PSD) The sample was prepared by diluting the original dispersion with ethanol or distilled water to 2000 times. The mean particle size and particle size distribution were determined at $25^{\circ} \mathrm{C}$ by a dynamic light scattering method (DLS, Malvern, England).

1.2 Optical and Polarized Optical Microscopy (OM/POM) Optical and polarized optical microscopy images were collected using polarized light with a sample between crossed polarizers by an inverted microscope (Sunny Optical Technology Co., Ltd, China). The images were taken with a color CCD camera (LUXOPTO Co., Ltd, China) under polychromatic illumination produced by a halogen lamp. All observations were carried out at an ambient temperature of $25^{\circ} \mathrm{C}$. Sandwiched optical cells were prepared using a microscope glass slide and a coverslip. The samples were contained in square wells on the glass slide and then covered by a coverslip.

1.3. Scanning Electron Microscope (SEM) The diameters and surface structure of the samples were examined by an SEM (SU1510, Hitachi, Co., Ltd, Japan) at an operation voltage of 5kV. The samples were sputter-coated with gold before the SEM observations.

1.4. Transmission Electron Microscope (TEM) The morphology of the samples was observed by a transmission electron machine (JEM-2100, Hitachi, Co., Ltd, Japan) under an operation voltage of $200 \mathrm{kV}$. The samples were diluted and dropped on a copper mesh (200 meshes) to allow the water to evaporate at room temperature.

1.5. Energy Dispersive Spectroscopy (EDS) The morphologies and surface element content of samples were characterized using an SEM apparatus (Hitachi su1510, Hitachi Co., Japan) with an energy dispersive spectrum (Axis supra, Kratos Co., UK).

1.6. Thermogravimetric Analysis (TGA) Thermal degradation of the samples was performed in the furnace of the TGA system (TGA-7, PerkinElmer, Co., Ltd, USA) operated under a nitrogen flow, heating from 30 to $800^{\circ} \mathrm{C}$ at the rate of $10^{\circ} \mathrm{C} / \mathrm{min}$.

1.7. Differential Scanning Calorimeter (DSC) The thermal characteristics of the samples were studied using a DSC (Q-200, TA Instruments, USA) in a nitrogen atmosphere. The samples were sealed in aluminum pans, and an empty sealed aluminum pan was used as a reference. For all DSC experiments, samples were first cooled down to $-10{ }^{\circ} \mathrm{C}$ and heated from -10 to $200{ }^{\circ} \mathrm{C}$, held isothermally for $5 \mathrm{~min}$ to remove thermal history, and then cooled to $-10^{\circ} \mathrm{C}$. The heating and cooling rates were $10{ }^{\circ} \mathrm{C} / \mathrm{min}$. Repeat this process twice. 
1.8. Fourier Transform Infrared (FT-IR) Spectra Fourier transform infrared (FT-IR) spectra were recorded on an FT-IR spectrometer (Nicolet is 10, Thermo Nicolet Co., Ltd, USA). A total of 32 scans for each sample were taken with a resolution of $8 \mathrm{~cm}^{-1}$.

1.9. Fiber-Optical Spectrometer (FOS) Putting the EM-DDLC-YS-Ms devices in FOS (FX-2000, Idea-optics Co., Ltd, China), and the EO responses were studied under an applied electric field using a function generator (Model Philips FG-8002). The reflectivity and transmittance value of EM-DDLC-YS-Ms change under the different applied voltage was recorded.

1.10 Heatable-Platform Polarizing Microscope (HP-POM) The phase transition characteristics of the samples were studied using an HP-POM (DM2700P, Leica microsystems, Germany). The samples were first cooled down to $30^{\circ} \mathrm{C}$ and heated from 40 to $90{ }^{\circ} \mathrm{C}$, held isothermally for 5 min to remove thermal history, and then cooled to $30{ }^{\circ} \mathrm{C}$. The heating and cooling rates were $1{ }^{\circ} \mathrm{C} / \mathrm{min}$. Repeat this process twice.

1.11. Synthesis Methods Through hydrophilic-hydrophobic interface switch emulsifier (Brij-35) formed a uniformly $\mathrm{O} / \mathrm{W}$ emulsion and following a simple seed interfacial polymerization strategy, a new breed of liquid crystals microcapsules with yolk-shell structure, included VTES-modified Fe3O4@SiO2 particle yolk, dye-doped liquid crystal albumen, and polymethylmethacrylate shell, termed as EM-DDLC-YS-Ms, was created.

Table S1. The physical properties of the liquid crystal E7.

\begin{tabular}{|c|c|c|c|}
\hline \multicolumn{4}{|c|}{ Liquid crystal E7 } \\
\hline \multirow[t]{2}{*}{ Refractive index $\left(25^{\circ} \mathrm{C} 589 \mathrm{~nm}\right)$} & $\mathrm{n}_{\mathrm{e}}$ & 1.746 & \multirow[t]{2}{*}{$\Delta \mathrm{n}=0.224$} \\
\hline & $\mathrm{n}_{\mathrm{o}}$ & 1.521 & \\
\hline \multirow[t]{2}{*}{ Permittivity } & $\varepsilon_{\|}$ & 19.5 & \multirow[t]{2}{*}{$\Delta \varepsilon=14.4$} \\
\hline & $\varepsilon_{\perp}$ & 5.1 & \\
\hline \multirow[t]{3}{*}{ Elastic constants $(\mathrm{pN})$} & & $\mathrm{K}_{11}$ & 11.7 \\
\hline & & $\mathrm{K}_{22}$ & 8.8 \\
\hline & & $\mathrm{K}_{33}$ & 17.1 \\
\hline Coefficient of viscosity $(\mathrm{mPa} \cdot \mathrm{S})$ & \multicolumn{3}{|c|}{100} \\
\hline
\end{tabular}

Chart S1. Composition and chemical structure of E7 and dichroic dye

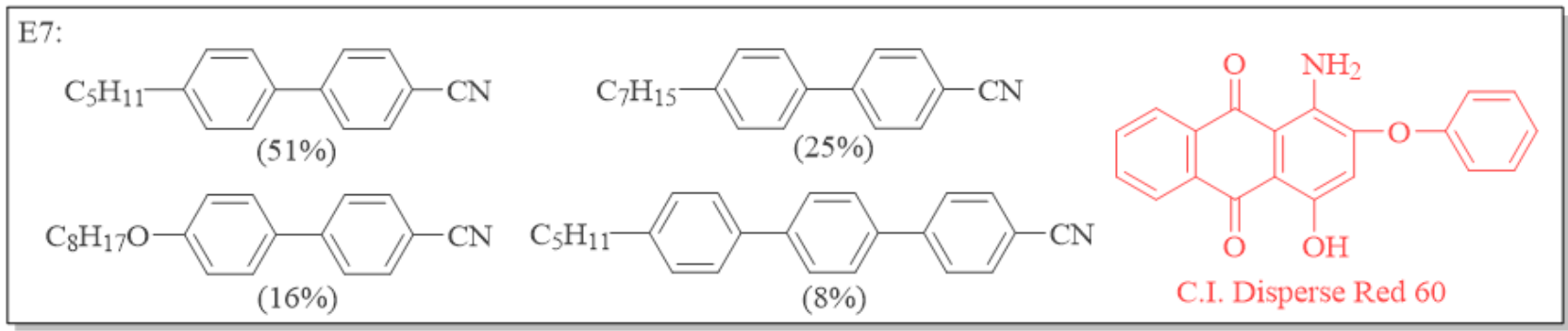




\section{Supplementary Results \& Discussion}

\subsection{Preparation and Characterization of Magnetic Seeds}
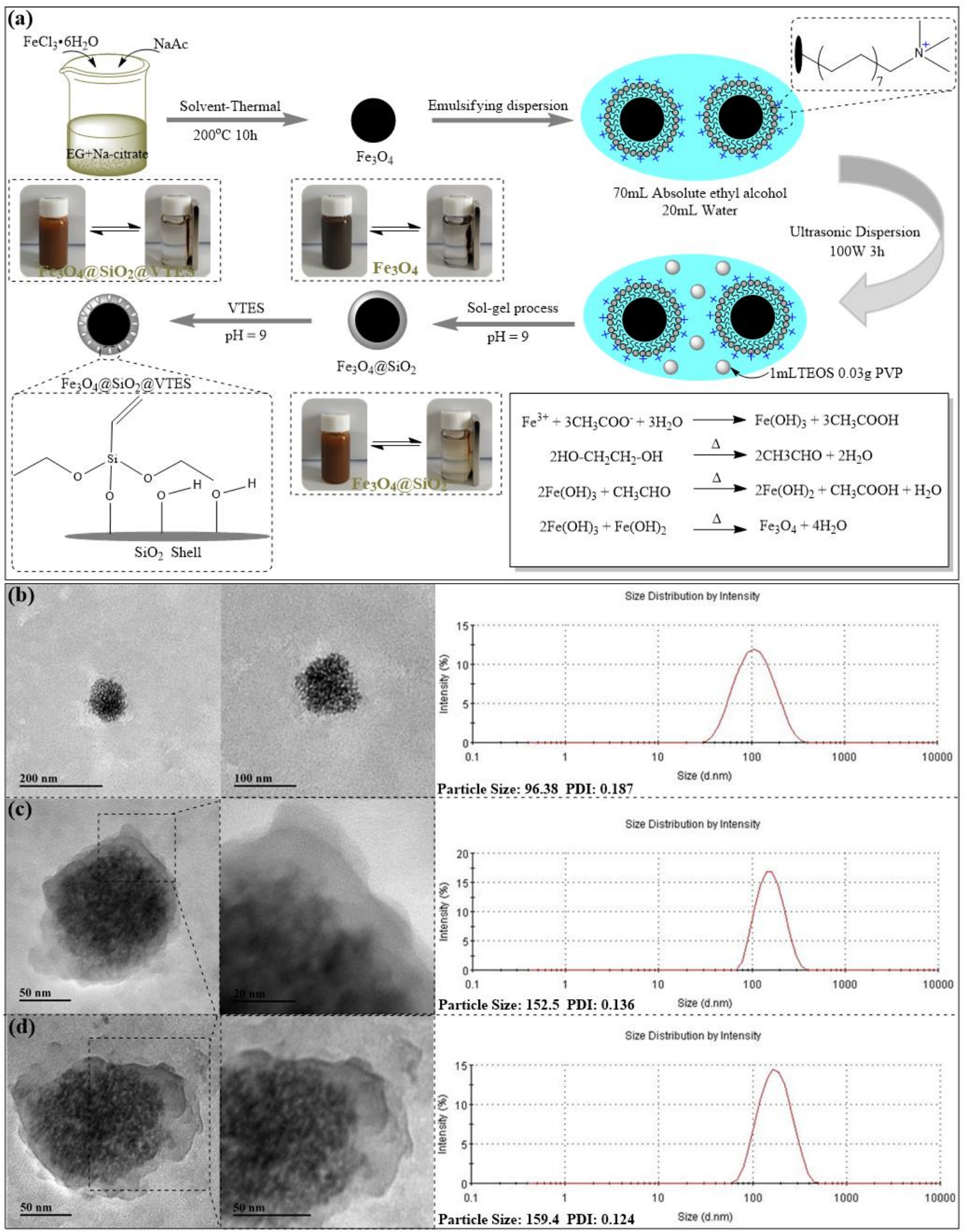

Figure S1. (a) Schematic illustration of the proposed formation mechanism and synthesis process of the magnetic seed, and the photograph of $\mathrm{Fe}_{3} \mathrm{O}_{4}, \mathrm{Fe}_{3} \mathrm{O}_{4} @ \mathrm{SiO}_{2}, \mathrm{Fe}_{3} \mathrm{O}_{4} @ \mathrm{SiO}_{2} @$ VTES dispersion with and without magnetic field; The TEM images and particle size distribution (PSD) of the modified (b) $\mathrm{Fe}_{3} \mathrm{O}_{4},(\mathrm{c}) \mathrm{Fe}_{3} \mathrm{O}_{4} @ \mathrm{SiO}_{2}$, and (d) $\mathrm{Fe}_{3} \mathrm{O}_{4} @ \mathrm{SiO}_{2} @$ VTES particles. 


\section{Note for Figure S1:}

To obtain the aforementioned microcapsule in the main-manuscript, the primary purpose is to synthesize the targeted magnetic seeds with excellent magnetic properties and suitable hydrophilic-hydrophobic properties, which fabrication procedure was depicted in Figure S1a. Firstly, water-dispersible $\mathrm{Fe}_{3} \mathrm{O}_{4}$ particles with spherical shape and uniform particle size $(\sim 96.4 \mathrm{~nm}$, Figure $\mathrm{S} 1 b)$ were synthesized by the solvothermal reaction. Subsequently, the monodispersed $\mathrm{Fe}_{3} \mathrm{O}_{4}$ particles were further coated with a protective shell of the silica layer $(\sim 56.1 \mathrm{~nm}$, Figure $\mathrm{S} 1 \mathrm{c})$ via the versatile sol-gel process using TEOS as a precursor. It has been reported that silica coating of the $\mathrm{Fe}_{3} \mathrm{O}_{4}$ particles can not only stabilize the particles effectively but also modify the surface properties, which favors the subsequent deposition of lipophilic VTES shells on the $\mathrm{Fe}_{3} \mathrm{O}_{4} @ \mathrm{SiO}_{2}$ templates based on the hydrolysis and coupling reactions. Therefore, the product (VTES-modified $\mathrm{Fe}_{3} \mathrm{O}_{4} @ \mathrm{SiO}_{2}$ ) is uniformly spherical with an average diameter of $\sim 159.4 \mathrm{~nm}$ as shown in Figure S1d. In particular, the obtained $\mathrm{Fe}_{3} \mathrm{O}_{4}$ particles can readily move in the water under the magnetic field and showed strong magnetic responsiveness, and the coating $\left(\mathrm{Fe}_{3} \mathrm{O}_{4} @ \mathrm{SiO}_{2}\right)$ and modification $\left(\mathrm{Fe}_{3} \mathrm{O}_{4} @ \mathrm{SiO}_{2} @ \mathrm{VTES}\right)$ process have little effects on its magnetic properties.

During the EM-DDLC-YS-Ms synthesis process, the key point is the uniform adsorption of DDLC/MMA on the surfaces of the magnetic seed. For water-dispersible $\mathrm{Fe}_{3} \mathrm{O}_{4}$ and $\mathrm{Fe}_{3} \mathrm{O}_{4} @ \mathrm{SiO}_{2}$ particles, the DDLC/MMA was difficult to be adsorbed on its surface in the emulsion due to the presence of a hydration layer formed by hydrophilic groups $(-\mathrm{OH})$ of particles. Conversely, there will be severe agglomeration due to the affinity of hydrophobic groups and magnetic attraction between them if the modified magnetic particles with highly hydrophobic. Herein, the graft amount of VTES on the surface of $\mathrm{Fe}_{3} \mathrm{O}_{4} @ \mathrm{SiO}_{2}$ particles was controlled in this study so that the surface of magnetic particles can become lipophilic and still maintain a certain dispersion in water. 

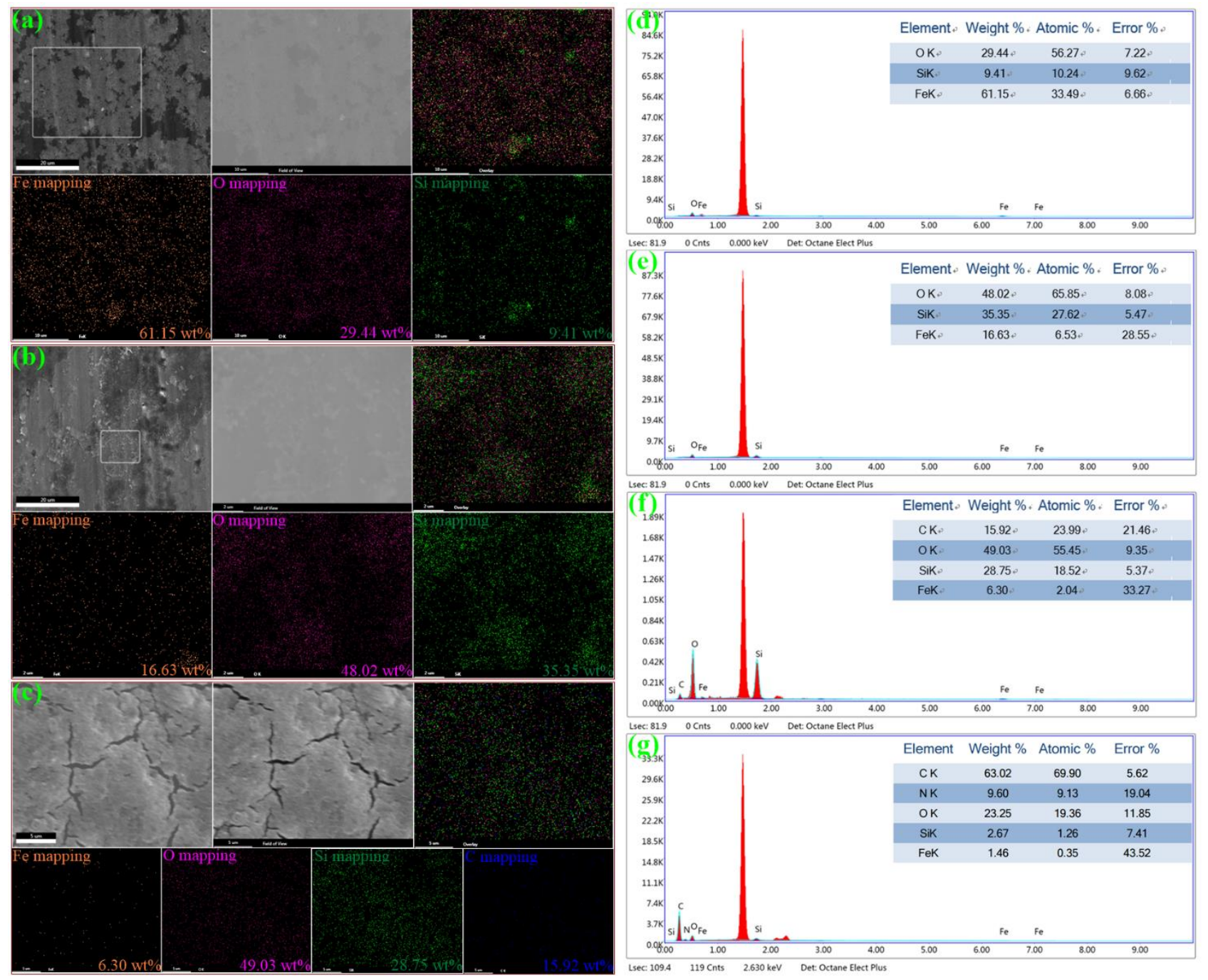

Figure S2. SEM-EDS mapping images of the synthesized (a) $\mathrm{Fe}_{3} \mathrm{O}_{4}$, (b) $\mathrm{Fe}_{3} \mathrm{O}_{4} @ \mathrm{SiO}_{2}$, (c) $\mathrm{Fe}_{3} \mathrm{O}_{4} @ \mathrm{SiO}_{2} @$ VTES particles. EDS analysis of the synthesized (d) $\mathrm{Fe}_{3} \mathrm{O}_{4}$, (e) $\mathrm{Fe}_{3} \mathrm{O}_{4} @ \mathrm{SiO}_{2}$, (f) $\mathrm{Fe}_{3} \mathrm{O}_{4} @ \mathrm{SiO}_{2} @ \mathrm{VTES}$, and (g) $\mathrm{Fe}_{3} \mathrm{O}_{4} @ \mathrm{SiO}_{2} @ \mathrm{VTES} @$ DDLC@PMMA particles.
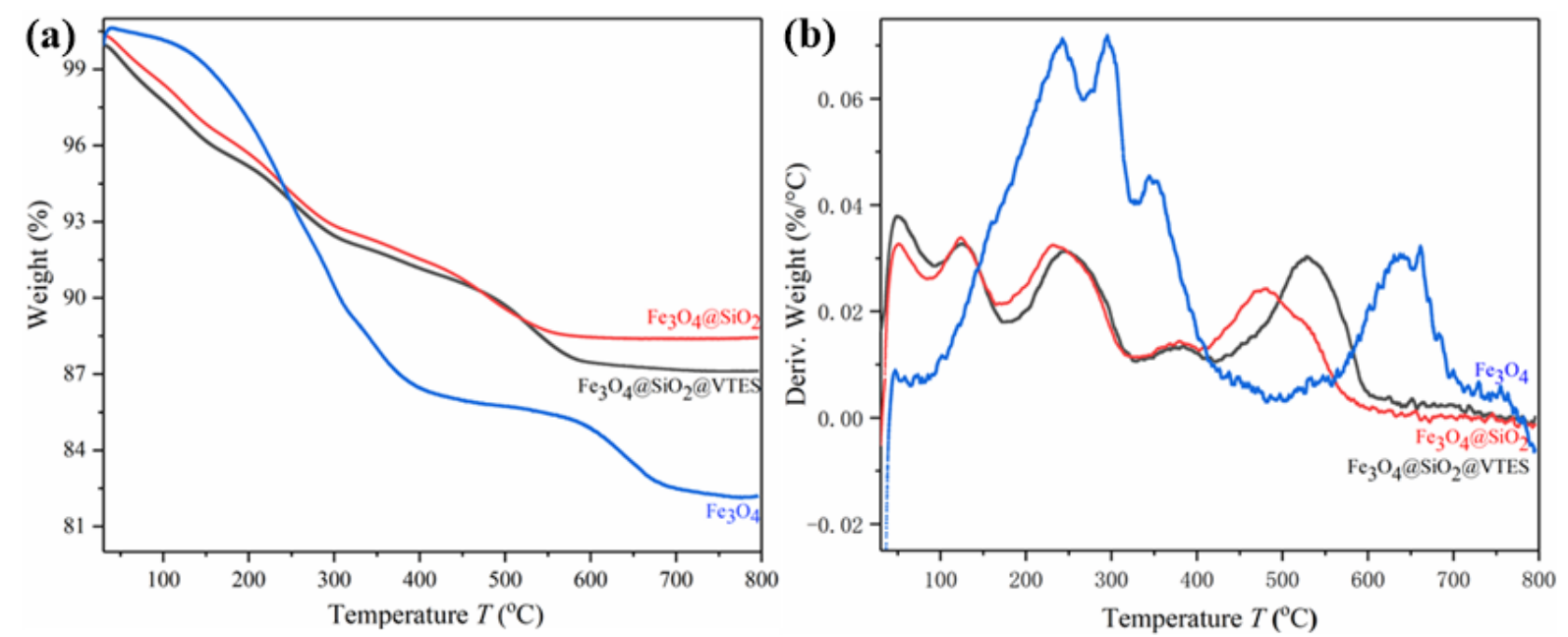

Figure S3. (a) TGA and (b) DTG analysis of the modified $\mathrm{Fe}_{3} \mathrm{O}_{4}, \mathrm{Fe}_{3} \mathrm{O}_{4} @ \mathrm{SiO}_{2}$, and $\mathrm{Fe}_{3} \mathrm{O}_{4} @ \mathrm{SiO}_{2} @ V T E S$ particles. 
2.2. Characterization and Performance of EM-DDLC-YS-Ms

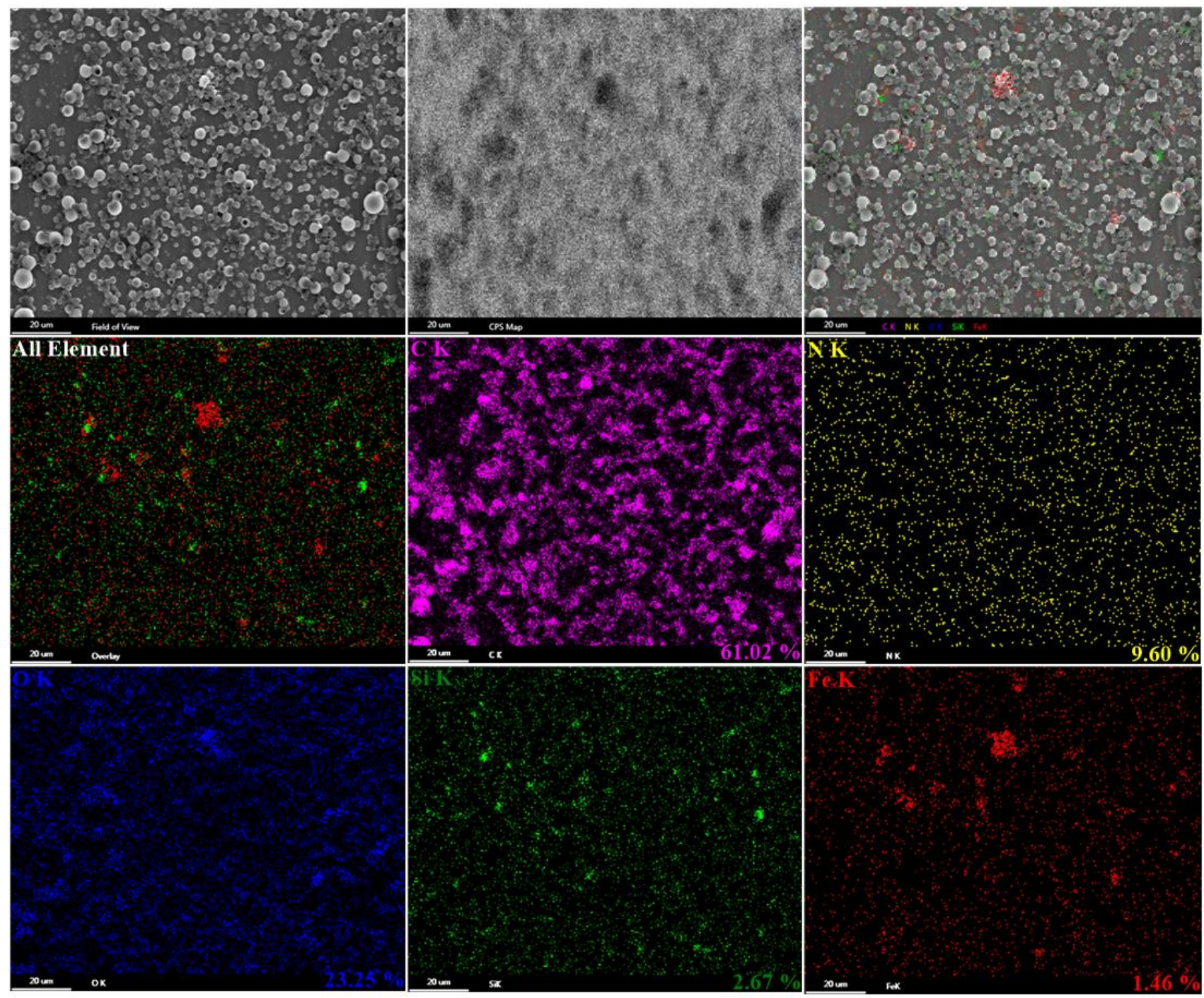

Figure S4. SEM-EDS mapping images of the EM-DDLC-YS-Ms. 


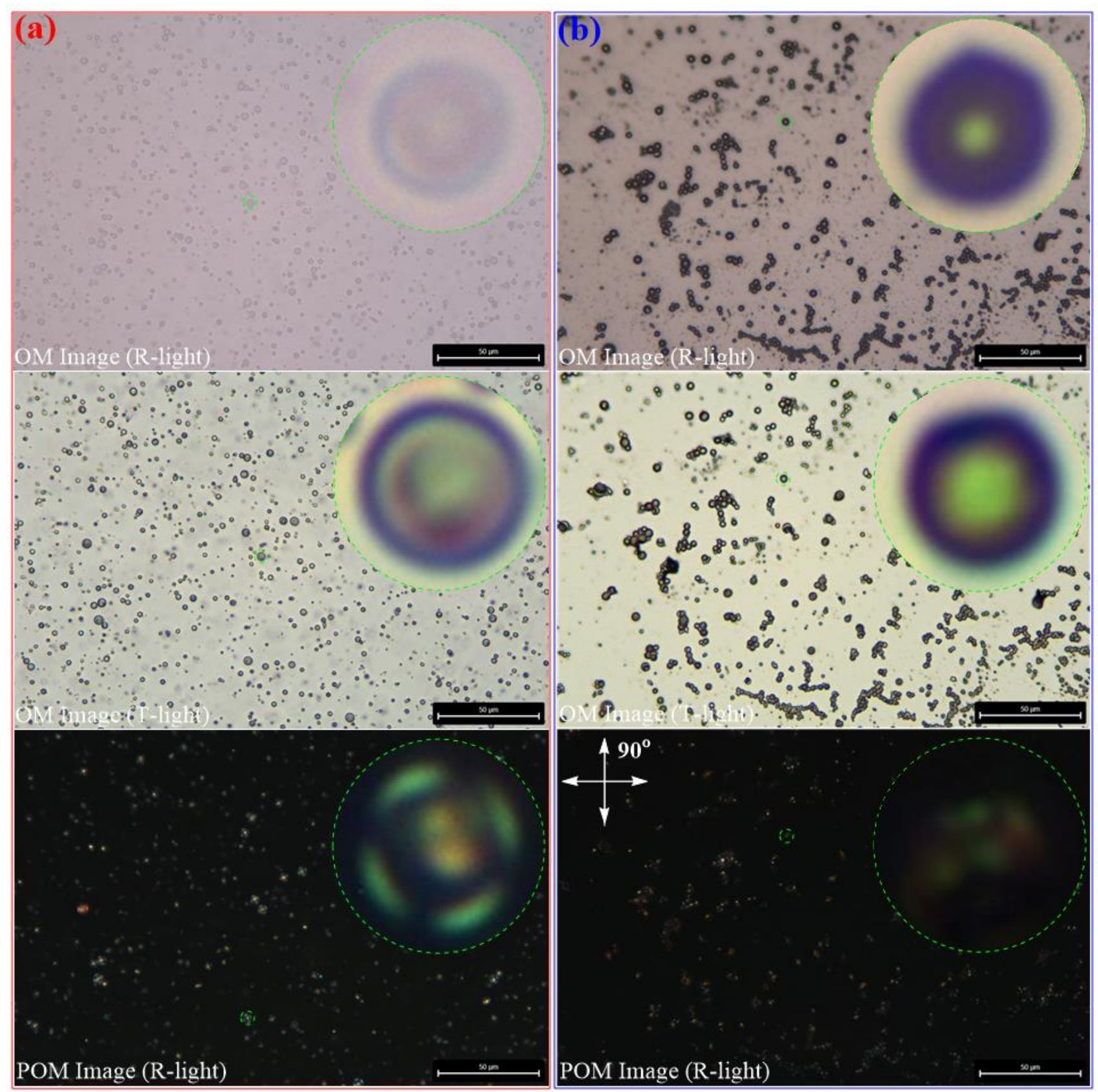

Figure S5. OM images (reflected light mode, R-light; transmitted light mode, T-light) and POM image (R-light) $(\times 500$ magnification) of the (a) EM-DDLC-YS-Ms emulsion and (b) dry synthesized EM-DDLC-YS-Ms particles. (scale bar, $50 \mu \mathrm{m}$ ) 

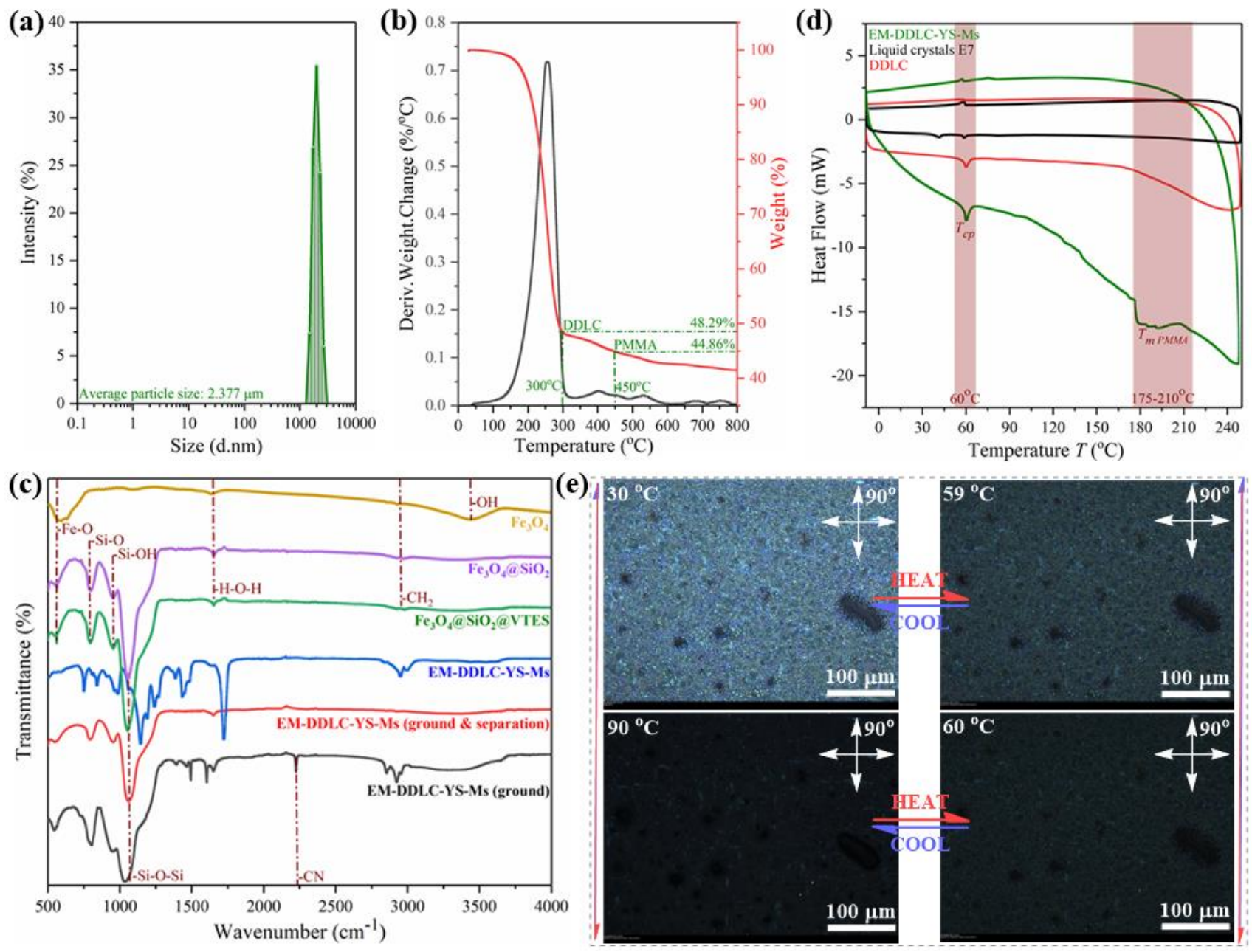

Figure S6. (a) PSD analysis and (b) TGA-DTG analysis of the synthesized EM-DDLC-YS-Ms particles; (c) FTIR-ATR and (d) DSC analysis of the EM-DDLC-YS-Ms; (e) HP-POM images of EM-DDLC-YS-Ms at different temperatures. 


\section{Note for Figure S6:}

FTIR-ATR was implemented to confirm DDLCs were enveloped in microcapsules (Figure S6c), and the penetration thickness of the IR beam is set to below $100 \mathrm{~nm}$. In the FTIR-ATR spectrum of DDLCs, a peak at $2225.85 \mathrm{~cm}^{-1}$ is attributed to nitrile bond $(-\mathrm{C} \equiv \mathrm{N})$ stretching. For DDLC-Ms, the DDLCs core is covered by different shell materials thicker than $250 \mathrm{~nm}$, and thus the characteristic peak of E7 at $2225.85 \mathrm{~cm}^{-1}$ could not be detected by the FTIR-ATR test. However, featured peaks belonging to E7 are observed in the spectra of vandalism of the DDLC-Ms, which means DDLCs material is successfully embedded in microcapsules. To acquire more detailed information about the effect of the yolk-shell structure on the property DDLC, the sample was put in HP-POM to measured its phase transition behavior. Based on previous research, a temperature shift for the phase transition of DDLCs is a result of different competing effects. In general, surface-induced ordering gives rise to an increased phase transition temperature, while elastic force-induced disordering reduces the phase

transition temperature. ${ }^{1-3}$ Here, the results indicated that the magnetic seed and PMMA shell have a little coefficient impact on the DDLCs order in EM-DDLC-YS-Ms as shown in Figure S6f and the same conclusion also can be drawn in DSC analysis (Figure S6d). 


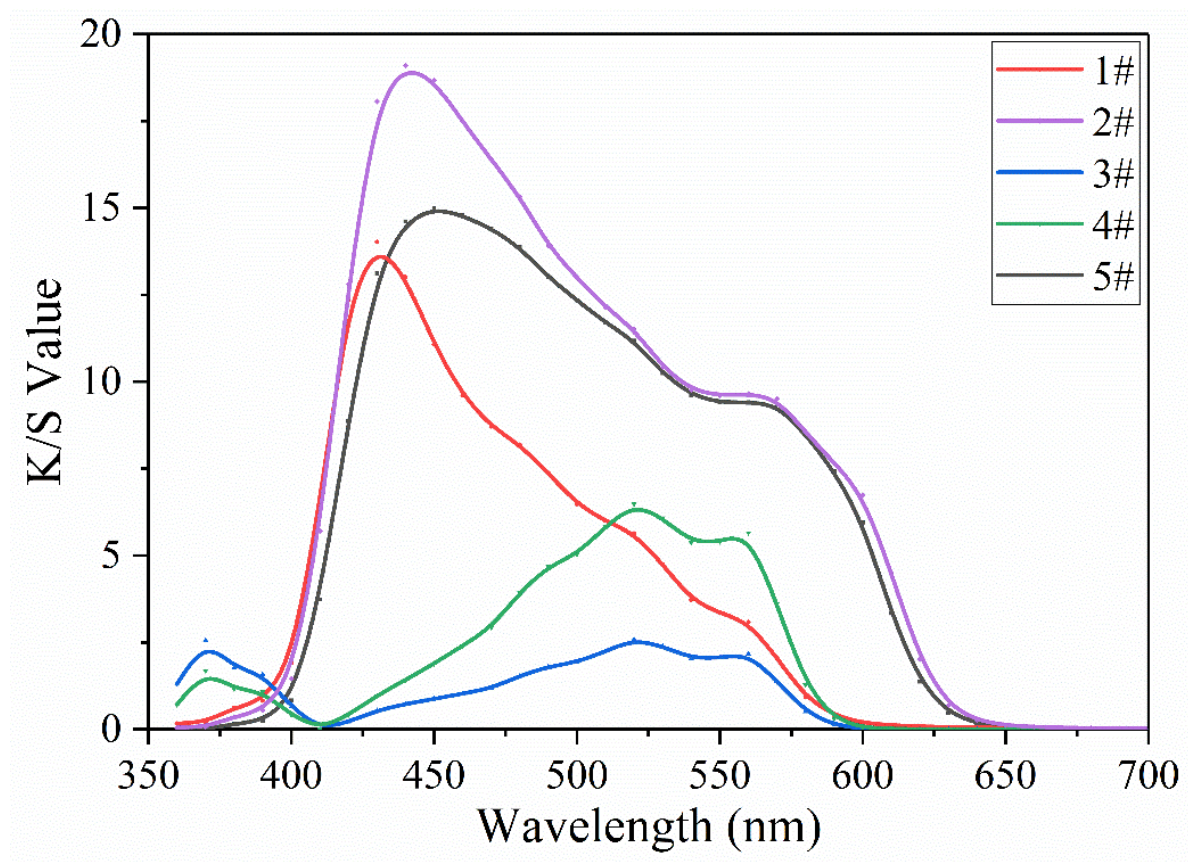

Figure S7. K/S value curve of dichroic dye (for 1\#), pure DDLC (for 2\#), DDLC emulsion (for 3\#), DDLC-Ms emulsion (for 4\#), and EM-DDLC-YS-Ms films (for 5\#).

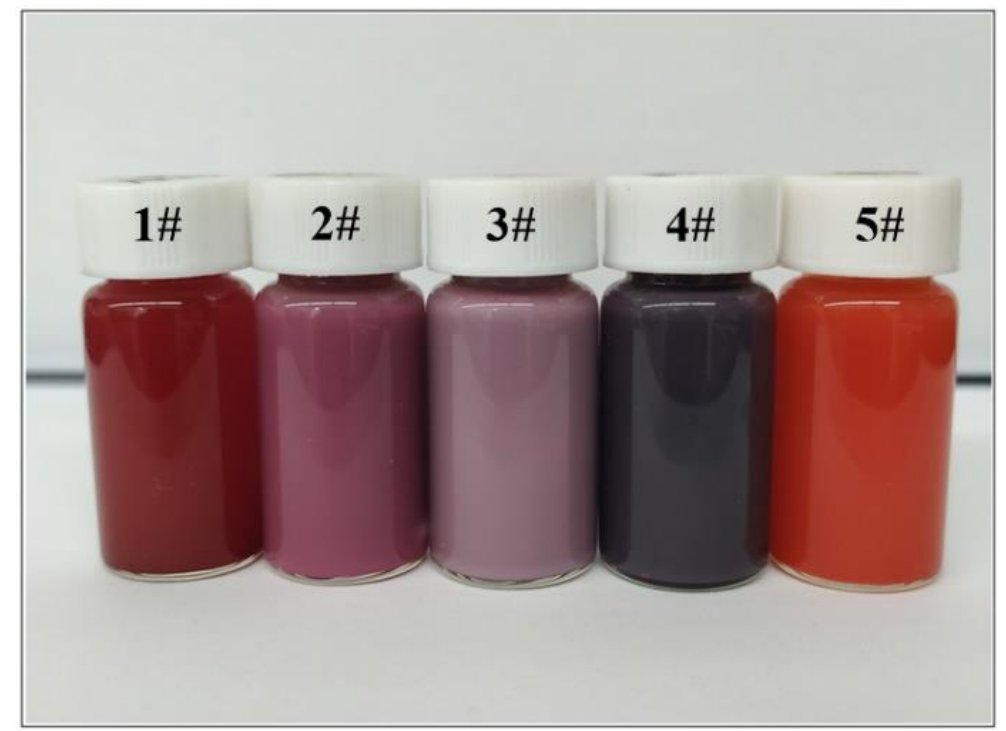

Figure S8. Photographs of pure DDLC (for $1 \#$ ), $\mathrm{Fe}_{3} \mathrm{O}_{4}$ particle doped DDLC emulsion (for 2\#), carbon black doped DDLC emulsion (for 3\#), black pigment paste doped DDLC emulsion (for 4\#), and EM-DDLC-YS-Ms emulsion (for 5\#). 


\section{Note for Figure S7 and Figure S8:}

In nature, non-iridescent structural plumage color is typically produced by coherent scattering of light within a matrix of cuticle and keratohyaline ("spongy layer") in feathers. For Cyanocitta stelleri, the underlying basal melanin layer under its spongy layer plays in the enhancement of coloration due to melanin functions to absorb incoherently scattered white light from the feather thereby increasing the purity of the color produced by the spongy layer. Interestingly, similar color enhancement effects have been observed in yolk-shell structures EM-DDLC-YS-Ms as shown in Figure 2a. Based on the characteristics of selective absorption of light by dichroic dyes, when the incident light shines the DDLC, the dyes absorb the specific wavelength of light, reflecting the complementary light to show the corresponding color. However, there exist complex differences in the optical property when DDLC was encapsulated in micelles or microcapsules. As shown in Figure 2a. in typical DDLC microcapsules, when natural light shines on the microcapsules, some part undergoes specular reflection, some part irradiation into the interior of the microcapsule followed with selectively absorbed and/or interior reflected and the rest part passes through the microcapsules repeating the above process, which caused a lot of complementary color lights into the view field and lead to hue and saturation of DDLC reduction. Conversely, there exist significant refractive index differences between yolk and shell space in the EM-DDLC-YS-Ms and resulting in the incident light can be sufficiently scattered. Besides, magnetic seed as an excellent absorbing material can effectively absorb complementary light and transmission light, improve the color saturation degree of DDLC.

To further verify the above conclusion, different emulsions (included the dichroic dye, DDLC, DDLC-Ms, EM-DDLC-YS-Ms) and pure DDLC with the same content of dichroic dye but used the raw material composition of EM-DDLC-YS-Ms were prepared. To digitize the different color performances of the above samples, the prepared emulsion was made into a dense film under the high press. The results showed that the K/S curve of EM-DDLC-YS-Ms film similar to the origin 
DDLCs film (Figure S7), which means that EM-DDLC-YS-Ms have good color reproduction performance. It was obvious that the hue of EM-DDLC-YS-Ms was closer to the origin dichroic dye (Figure $2 \mathrm{~b}, 2 \mathrm{c}$ ), and the transmittance and $\mathrm{K} / \mathrm{S}$ value analysis (Figure $\mathrm{S} 7$ ) also indicted that EM-DDLC-YS-MS have good hue reproduction performance.

To further verify the color enhancement effect of the EM-DDLC-YS-Ms with black absorbent as yolk in the core-shell structure, the corresponding comparative experiment was performed as shown in Figure S8. In particular, the $\mathrm{Fe}_{3} \mathrm{O}_{4}$ (nano-powder, $20 \mathrm{~nm}$, Beijing Innochem Technology Co., Ltd.), carbon black (nano-powder, $100 \mathrm{~nm}$, Shanghai Cai-Ming Chemical Co., Ltd.), and black pigment paste (M-200, Shanghai Cai-Ming Chemical Co., Ltd.) were simply doped into DDLC emulsion as an absorbent. It can be seen that the doping of the black substrate significantly reduces the scattering phenomenon of the entire DDLC emulsion system. However, the pure mixing method will cause the color of the entire system to tend to be black, and thus damaging the hue of the DDLC itself (see in 2\#, 3\#, 4\# of Figure S8). 


\subsection{Supernumerary Photoelectric Performance of EM-DDLC-YS-Ms}
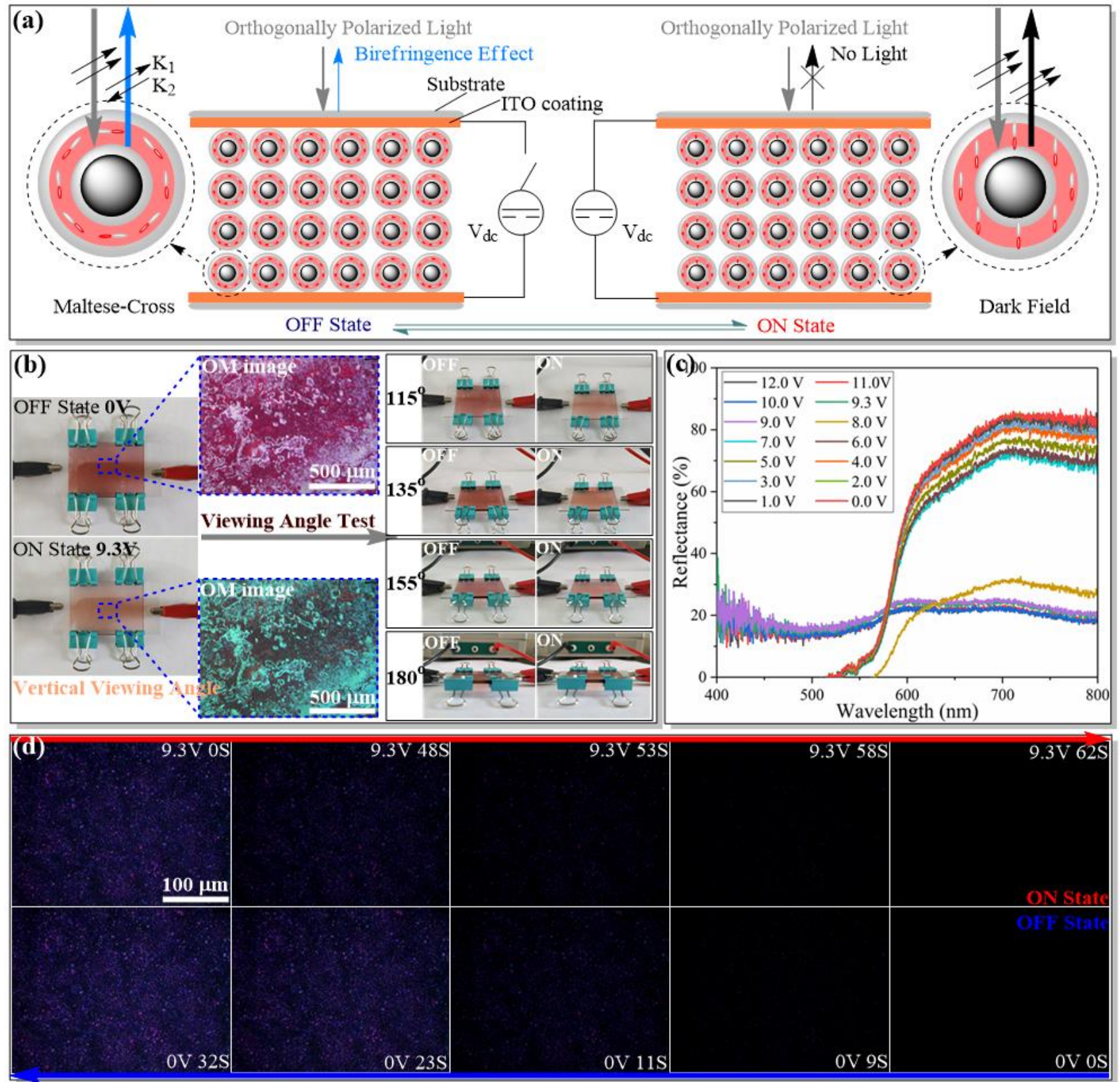

Figure S9. (a) The schematic of the EO property of EM-DDLC-YS-Ms measurements under POM. (b) Photographs, OM images, and viewing angle test images of EM-DDLC-YS-Ms device with applied and removed voltage. (c) Wavelength dependence of the reflectance of EM-DDLC-YS-Ms device under the different applied voltage. (d) POM images of EM-DDLC-YS-Ms dynamic change by applied and removed voltage. 


\section{Note for Figure 3 and Figure S9:}

To observe and analyze the photoelectric performance of microcapsules conveniently, the microcapsules were filled in the cells which were made of two pieces of thin indium tin oxide (ITO) glasses, and subsequently, the color variation and polarized light pattern could be observed with applied voltage changed as shown in Figure 3a. According to the electric field response characteristics of nematic liquid crystals, the optic axis of DDLC was oriented in the same direction in the presence of voltage, and the visual field changed from bright light to completely dark due to the influence of DDLC arrangement on the optical path depicted in Figure S9a. Herein, the driving voltage $\left(E_{t h}\right)$ was defined as the voltage that can deflect the DDLC completely, the response time $\left(\tau_{r}\right)$ as the time when the DDLC in the microcapsule completely deflects with the electric field, and delay time $\left(\tau_{d}\right)$ as the time required for the DDLC in the microcapsule to recover after the electric field is removed. EM-DDLC-YS-Ms were placed under POM by supplying voltage to explore the limited behavior of DDLC in EM-DDLC-YS-Ms. Figures 3d and S9d shown that the driving voltage, response time, and delay time of EM-DDLC-YS-Ms were $9.3 \mathrm{~V}, 62 \mathrm{~s}$, and $32 \mathrm{~s}$, respectively, and which may be attributed to the DDLC anchored by the spherical inner surface of the microcapsule and/or magnetic seed to stunted the rotation of the optic axis. 


\subsection{Demo of Recyclable Process of EM-DDLC-YS-Ms Film}

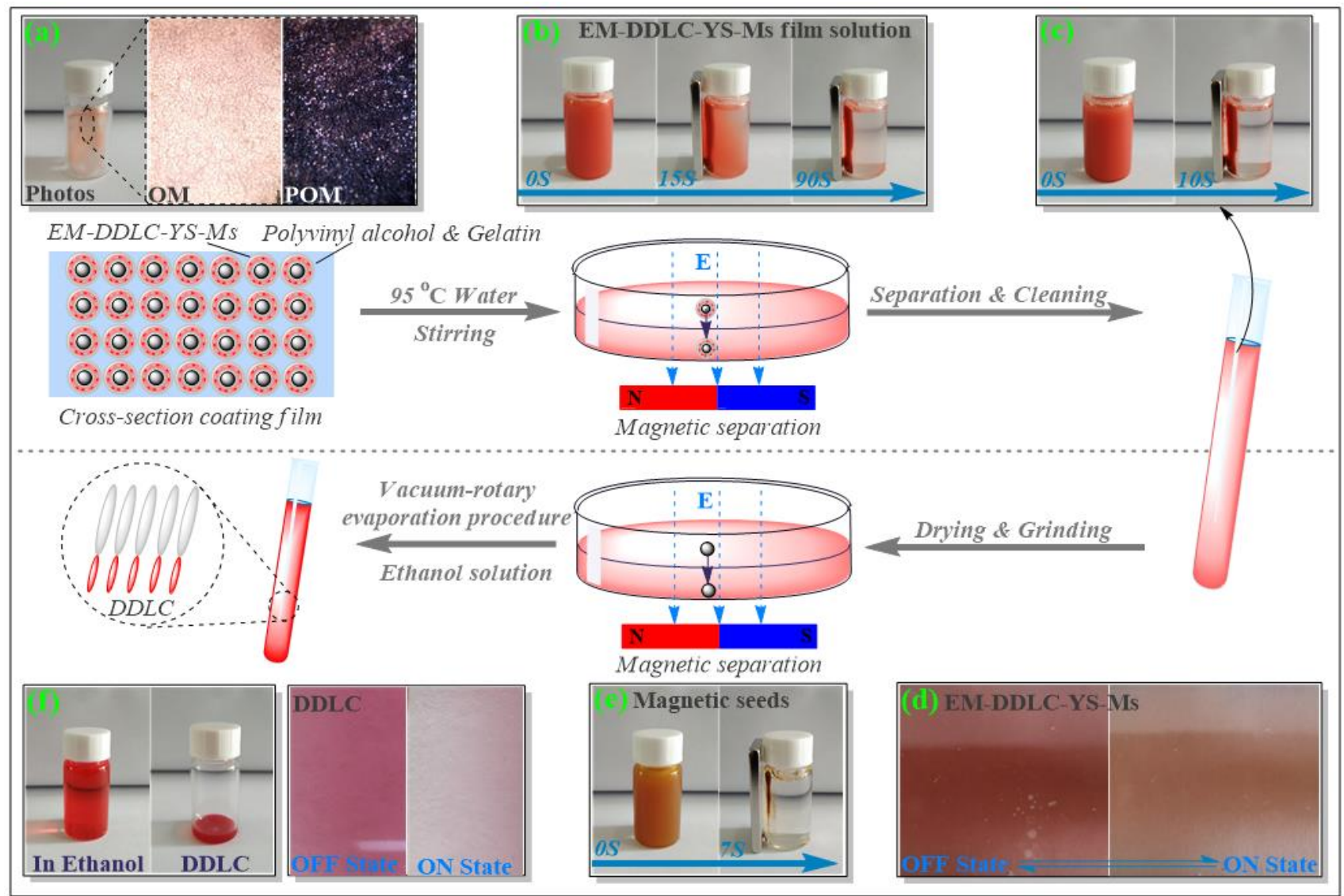

Figure S10. Schematic illustration and photographs of the proposed recyclable process for EM-DDLC-YS-Ms: (a) the photograph, OM image, and POM image of EM-DDLC-Ms-YS-Ms film; magnetic separation demo of the (b) EM-DDLC-YS-Ms film solution, (c) EM-DDLC-YS-Ms emulsion and (e) magnetic seeds from ground microcapsules; (d) the demonstration of electrical stimulation response process of (e) separated EM-DDLC-YS-Ms and (f) purified DDLC by ethanol.

\section{Note for Figure S10:}

Traditional development and design for DDLC material mainly rely on liquid crystal cell, polymer dispersed liquid crystal, and microcapsules technology, which lacks effective DDLC recycling technology leads to waste of resources and environmental pollution. According to the above research results in the main manuscript, an idea of enrichment-separation of waste EM-DDLC-YS-Ms based production was proposed as shown in Figure S10. The printing paste was formulated including EM-DDLC-YS-Ms emulsion (35 wt\%), polyvinyl alcohol \& gelatin (10 wt $\%)$, and distilled water (55 wt $\%$ ). The PET-ITO glass was fixed on a smooth platform and the scraping speed was optimized to make a homogeneous coating with a thickness of $80 \mu \mathrm{m}$. Subsequently, the coating was dried at 
$80{ }^{\circ} \mathrm{C}$ for $10 \mathrm{~min}$ and then cured at $100{ }^{\circ} \mathrm{C}$ for $3 \mathrm{~min}$. The printing coating was further sandwiched in cells which were made of two pieces of PET-ITO films to prepare the flexible EM-DDLC-YS-Ms device. To demonstrate the details of the recovery process, the EM-DDLC-YS-Ms coating film was mechanically removed from the device and place in a sample bottle follow by hot water and stirring at high speed to dissolve the PVA-Gelatin film (Figure S10a). Subsequently, the concentrated EM-DDLC-YS-Ms emulsion was obtained by repeat magnetic separation and cleaning process from the EM-DDLC-YS-Ms film solution, which showed faster magnetic response property (Figures S10b, c). In particular, the obtained EM-DDLC-YS-Ms still keep good electrochromic properties when it was encapsulated in an ITO glass cell under the electric field as shown in Figure S10d. Then, as previously mentioned EM-DDLC-YS-Ms were dried, crushed, and further dissolved in water, and thus magnetic seeds were obtained under multiple magnetic separation processes as shown in Figure S10e. Finally, ethyl alcohol was introduced into the system and repeated extracted and purified several times to prepare DDLC/ethanol mixture, and then pure DDLC was obtained through vacuum drying. Besides, the excellent electrochromic performance of obtained DDLC was also demonstrated as shown in Figure S10f. 


\subsection{Supernumerary Magnetically Controllable Performance of EM-DDLC-YS-Ms}

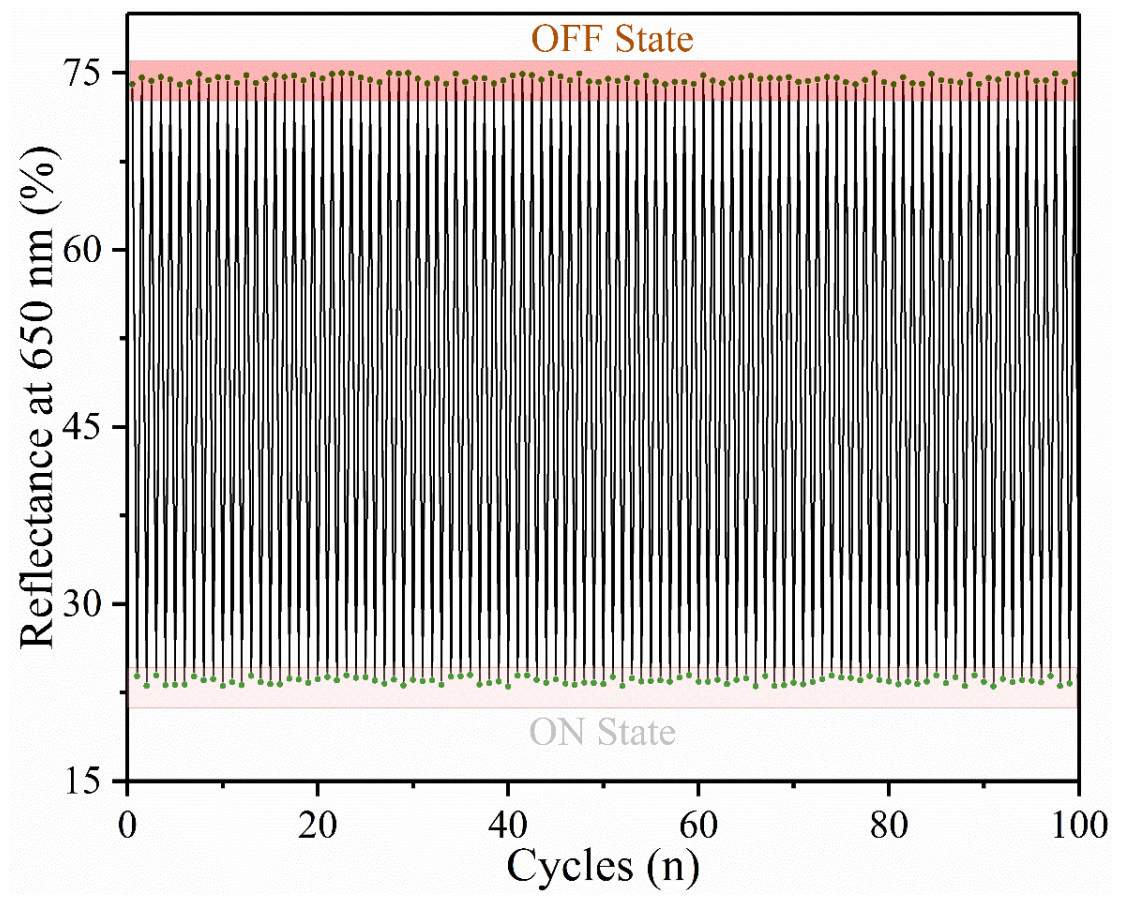

Figure S11. Maximum reflectance of the magnetically controlled EM-DDLC-YS-Ms device at 650 nm during the applied and removed electric field cycles.

\section{Note for Figure 4 and Figure S11:}

The EM-DDLC-YS-Ms particle dispersion fluid can be still oriented movement by a magnet as shown in Figure $4 \mathrm{~b}$, and its magnetic properties were also investigated using a superconducting quantum interference device (SQUID) magnetometer. Although the microencapsulation effect reduces the crystallinity of ferroferric oxide (Figure 4c), the EM-DDLC-YS-Ms particle dispersion fluid can be still oriented movement by a magnet as shown in Figure $4 \mathrm{~b}$. Hysteresis loops of the samples measured at $300 \mathrm{~K}$ shown no remanence or coercivity, suggesting a superparamagnetic character, and the saturation magnetization $(M s)$ values of EM-DDLC-YS-Ms are determined to be $12.78 \mathrm{emu} / \mathrm{g}$ as shown in Figure 4d. Thus, the magnetically controlled EM-DDLC-YS-Ms device was assembled by using a miniature magnet array to demonstrate the specific applicability of the EM-DDLC-YS-Ms as shown in Figure 4a. The EM-DDLC-YS-Ms-based coating was prepared by dispersing the monodispersed $\mathrm{SiO}_{2}$ microsphere with an average diameter of about $10 \mu \mathrm{m}$ in 
prefabricated EM-DDLC-YS-Ms concentrated emulsion. One drop of the above coating dispersion was poured on the center of a clean ITO glass plate and then a second clean ITO glass plate was carefully placed on top and slightly compressed following regular circular movements until the particles are in contact with both glass plates to obtained the magnetically controlled EM-DDLC-YS-Ms prototype device as shown in Figures $4 \mathrm{a}$ and $4 \mathrm{e}_{\mathrm{I}}$. The presence of $\mathrm{SiO}_{2}$ microspheres allows the EM-DDLC-YS-Ms to move freely within the cell. Therefore, the cell was exposed to a miniature magnet array which was arranged into specific characters (“JNU”, Figure 4e $\left.\mathrm{e}_{\text {II }}\right)$ for 15 min to make the EM-DDLC-YS-Ms form corresponding characters (Figure 4e magnetic field. Subsequently, the device was operated by supplying voltage from Direct Current (DC) power supply. In the absence of an electric field, DDLC was randomly distributed, and "JNU" in the cell can be seen in naked eyes main with the color of the dye. Applying an electrical field, the alignment of DDLC will parallel to the electric field result in the words "JNU" change to the fade-color state as shown in Figure 4e $\mathrm{e}_{\mathrm{IV}}$. Further FOS analysis also indicated that the reflectance of the EM-DDLC-YS-Ms device significantly decreases from $74.70 \%$ to $23.58 \%(650 \mathrm{~nm})$ under driving voltage $(\sim 9.7 \mathrm{~V})$ as shown in Figure $4 \mathrm{f}$. Meanwhile, the reflectance of contrast device without doped EM-DDLC-YS-Ms (termed as Blank Space) almost no change under the electric field. In particular, the emerging shock waves in the reflection curve were probably caused by the cavity effect due to the existence of $\mathrm{SiO}_{2}$ microspheres. Moreover, the visible light spectra of the device at 0 $\mathrm{V}$ and $9.7 \mathrm{~V}$ under 100 test cycles were almost identical to the initial maximum reflectance spectrum $(650 \mathrm{~nm})$ as shown in Figure S11, manifesting the long-term stability of the as-made device. In conclusion, EM-DDLC-YS-Ms showed its unlimited potential in the field of smart windows. 

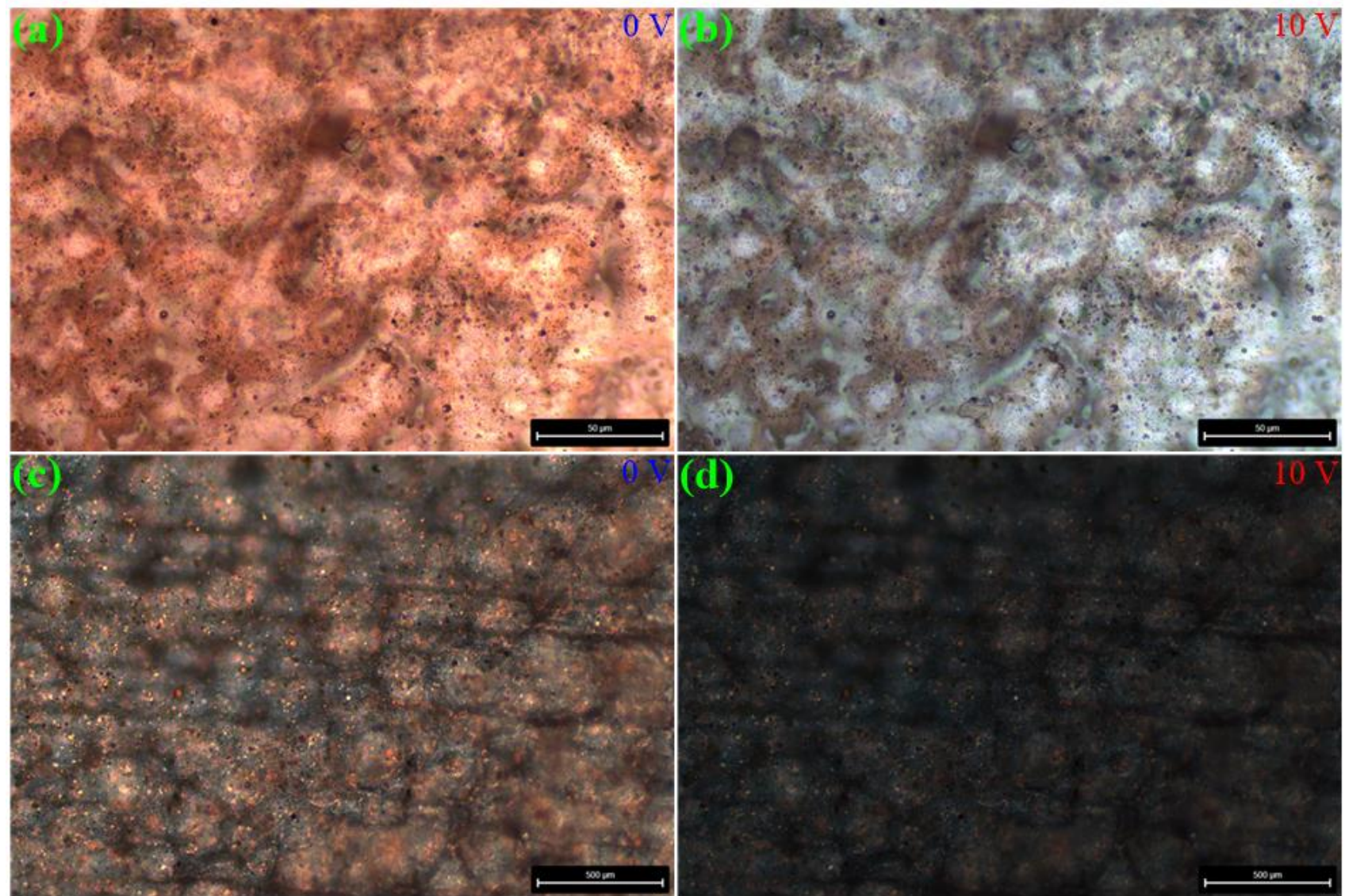

Figure S12. OM/POM images demonstrating the reversible change of the as-made EMs-Fabric in response to the electric field: the OM images of (a) OFF State and (b) ON State, and POM image of (c) OFF State and (d) ON State (reflected light mode, R-light) $(\times 500$ magnification). 

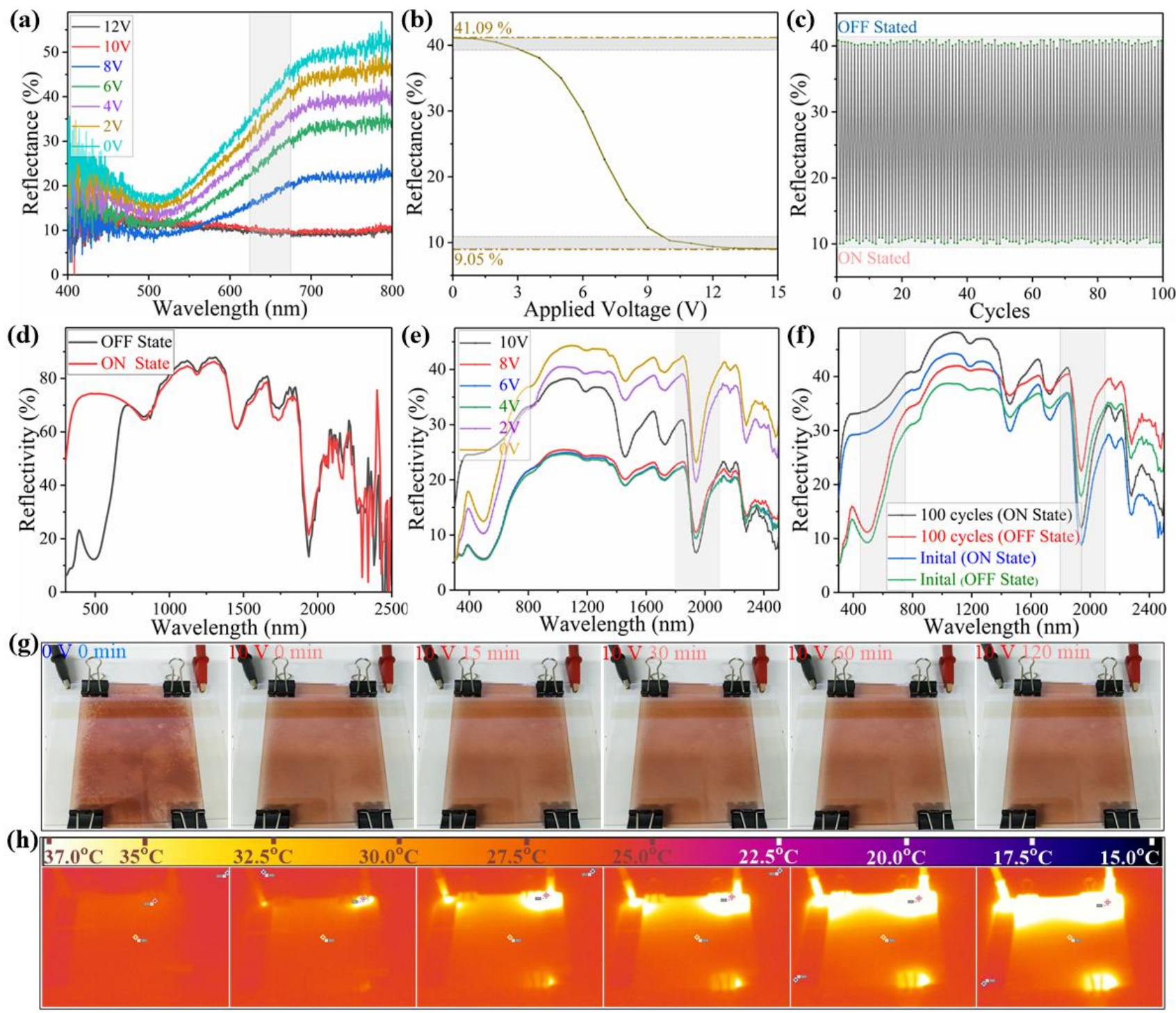

Figure S13. (a) Reflectivity curve of the EMs-Fabric with various voltages applied. (b) Voltage dependence of the reflectance for the intelligent fabric device. (c) Maximum reflectance of the EMs-Fabric device at $650 \mathrm{~nm}$ during the applied and removed electric field cycles. (d) The comparison of the Vis-NIR transmittance spectra of the DDLC-Ms non-woven fabric device (prepared by the same method as the EM-DDLC-YS-Ms non-woven fabric device but without the magnetic seeds) under on/off state $(0 \mathrm{~V} / 10 \mathrm{~V})$. (e) Vis-NIR reflectivity curve of the EMs-Fabric with various voltages applied. (f) Reflectance spectra of the film at the initial state and after 100 cycles. (g) Photographs, and (h) infrared thermic images of the EMs-Fabric device under applied voltages with time prolonged (recorded at a distance of $20 \mathrm{~cm}$ from the device). 


\section{Note for Figure 5, Figure S12, and Figure S13:}

As schematically depicted in Figure 5a, multi-spectral electromagnetic wave response EM-DDLC-YS-Ms non-woven-fabric, termed as EMs-Fabric, were designed according to the following criteria: (i) microcapsules must be evenly dispersed in the spinning solution to avoid clogging the nozzle; (ii) the accumulated PVP nano-fibers should form interconnected channels with small pore size to resist movement of microcapsules and keep high optical transparency. The electrospinning apparatus included a high-voltage supply (Dongwen Co., Tianjin, China), a micro-infusion pump (Medical Instrument Co., Zhejiang, China), a $20 \mathrm{~mL}$ syringe with a metal needle, and aluminum foil as the collector. Each solution was drawn horizontally from the needle tip by the electrostatic force generated from the high voltage applied between the tip and the collector. It formed a Taylor cone and jetted through the needle tip to the collector. Figure $5 \mathrm{~b}$ reveals that the prepared EMs-Fabric with dark red appearance still has a birefringence effect under polarizing light. SEM-EDS analysis (Figures 5c, 5d) indicated that the microcapsules were densely and evenly wrapped by crosslinked PVP nano-fiber.

EMs-Fabric can reversibly change from dark red (bright state, POM) to gray (dark state, POM) as the voltage applied and removed as shown in Figure S12, suggesting that the optic axis of the DDLC layer still keep reorientation capability even encapsulated in the fabric. Figure S13a, 13b shows that the device has reflection peaks (around 620-740 nm) attributed to DDLC in its initial state, and it exhibits a dark red color under visible light. As the applied voltage increases from 0 to $10 \mathrm{~V}$, red light reflectivity decreases from $41.1 \%$ to $10.1 \%$ caused by the orientation of DDLC. Subsequently, the red color fades to colorless and shows the gray color caused by PVP nano-fiber and magnetic seeds. Moreover, the cycles of applying/removing the electric field can be repeated at least 100 times, and the maximum reflectivity of the fabric has hardly changed (Figure S13c), which indicates that the device has good electrochromic stability. 
Since $\mathrm{Fe}_{3} \mathrm{O}_{4}$ is a medium-inverse spinel-type ferrimagnetic material, it can generate magnetic losses and electrical losses to electromagnetic waves. Thus, as-made EMs-Fabric is also a double complex dielectric material that has absorption properties for near-infrared microwaves. The vis-NIR spectra of the devices (Figure S13d, 13e) indicated that the applied voltage mainly decreased the reflectivity in the visible light region, but the Vis-NIR light could still be effectively blocked due to the absorption performance of the $\mathrm{Fe}_{3} \mathrm{O}_{4} @ \mathrm{SiO}_{2} @$ VTES core of the microcapsules. Moreover, the vis-NIR spectra of the device before and after the cycles were compared (Figure 13f). The vis-NIR spectra of the device at $0 \mathrm{~V}$ and $10 \mathrm{~V}$ after 100 test cycles were almost the same as the initial spectra, indicating the long-term stability of the as-made device.

Figures $13 \mathrm{~g}$ and $13 \mathrm{~h}$ show the relationship within the generated temperature, the real-color of the intelligent fabric device, and the time of voltage applied. It can be found that the color depth of an intelligent fabric device decreases obviously when the applied voltage is $10 \mathrm{~V}$, but almost no longer change with further prolonged time. Meanwhile, the heating phenomenon of the fabric (non-electrode contact position) was not significant (compared with body temperature, $\sim 37^{\circ} \mathrm{C}$ ) when it is energized for a long-time $(\sim 2 \mathrm{~h})$ as shown in Figure $\mathrm{S} 13 \mathrm{~h}$, which show its potential application prospect in visible light and infrared camouflage. 

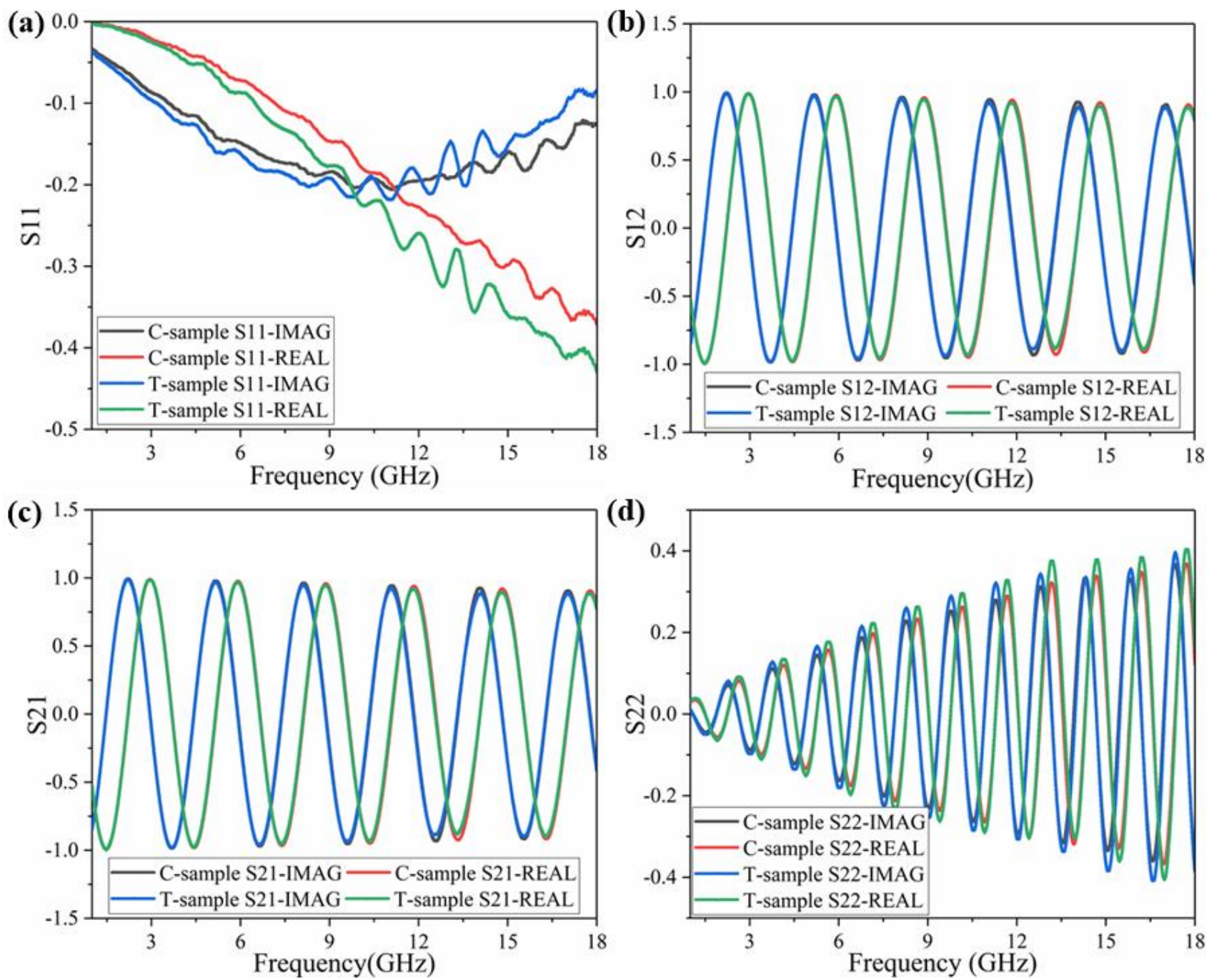

Figure S14. The comparison of the $S$ parameter (a) $S_{11}$ (b) $S_{12}$; (c) $S_{21}$; (d) $S_{22}$ of electromagnetic shielding sample (EM-DDLC-YS-Ms non-woven fabric for T-sample, and the DDLC-Ms non-woven fabric prepared by the same method as the EM-DDLC-YS-Ms non-woven fabric device but without the magnetic seeds for C-sample). 

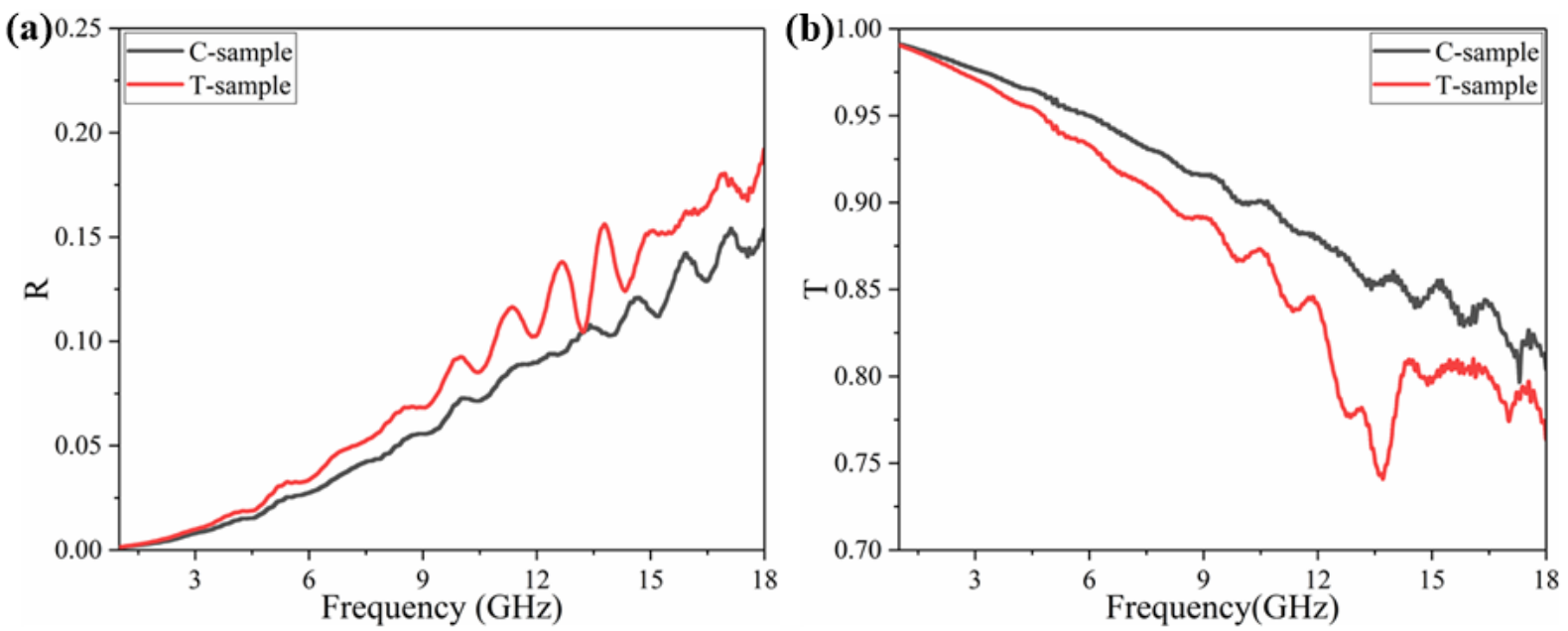

Figure S15. The comparison of the (a) $R$ and (b) $T$ parameters of electromagnetic shielding sample (EM-DDLC-YS-Ms non-woven fabric for T-sample, and the DDLC-Ms non-woven fabric prepared by the same method as the EM-DDLC-YS-Ms non-woven fabric device but without the magnetic seeds for C-sample).

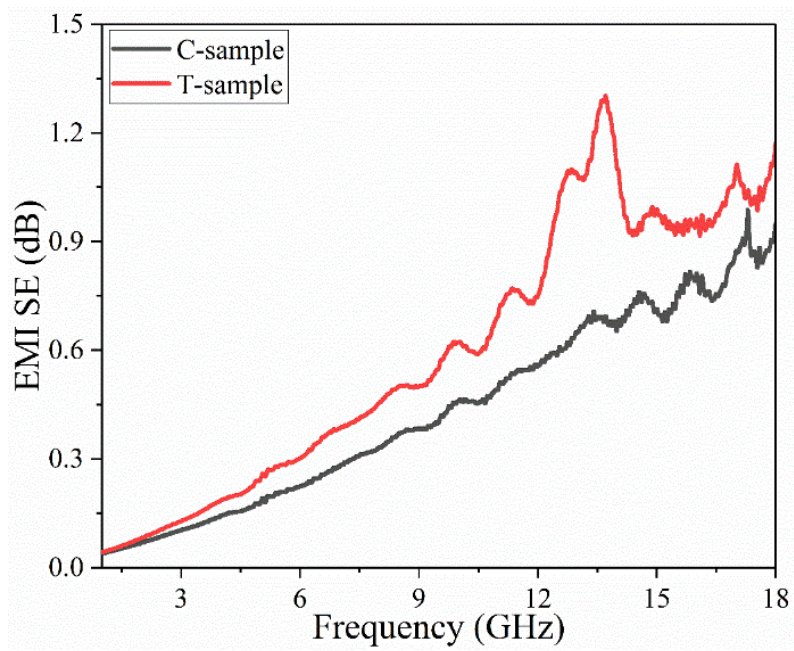

Figure S16. The comparison of the $S E_{T}$ parameters of electromagnetic shielding sample (EM-DDLC-YS-Ms non-woven fabric for T-sample, and the DDLC-Ms non-woven fabric prepared by the same method as the EM-DDLC-YS-Ms non-woven fabric device but without the magnetic seeds for C-sample). 


\section{Note for Figure S14, Figure S15, and Figure S16:}

In this experiment, the S-parameters of the samples were tested with a microwave network analyzer model PNA-N5244A E5071C (Agilent Co., Ltd, USA). The non-woven fabric fragments (using the microcapsules prepared by the same process with and without magnetic seed particles) and paraffin are mixed evenly according to the mass ratio of $1: 2$, heated to disperse the paraffin after melting and pressed into the mold to make a ring sample. The sample size was $7 \mathrm{~mm}$ in outer diameter, $3 \mathrm{~mm}$ in inner diameter, and $2 \mathrm{~mm}$ in thickness, which was marked as the test sample (T-sample) and comparative sample (C-sample), respectively. The S-parameters of the samples were measured by the coaxial method, and the test frequency range was 1-18 GHz. Therefore, the S parameter of the electromagnetic shielding samples was shown in Figure S14.

When the electromagnetic wave is incident on the shielding material, it will be divided into three parts: part of it is absorbed $(A)$, a part is reflected $(R)$ which included surface reflection and internal multiple reflections, and the other part is transmitted $(T) . R, T$, and $A$ following the relationships exist:

$$
\begin{gathered}
I=A+R+T \\
A=\frac{P_{a}}{P_{i}} \\
R=\frac{P_{r}}{P_{i}}=\left|S_{11}\right|^{2} \\
T=\frac{P_{t}}{P_{i}}=\left|S_{21}\right|^{2}
\end{gathered}
$$

Among them, $P_{i}, P_{a}, P_{r}$, and $P_{t}$ represent the energy of the incident wave, absorption wave, reflection wave, and transmission wave. $S_{11}$ and $S_{12}$ are $S$ parameters. Therefore, the $R$ and $T$ parameters of the electromagnetic shielding sample were shown in Figure S15. 
Electromagnetic shielding effectiveness (EMI SE) was used to indicate the shielding effectiveness of electromagnetic shielding materials. The shielding effectiveness $S E_{T}$ is the sum of absorption shielding effectiveness and reflection shielding effectiveness, which can be calculated by the following formula: ${ }^{4}$

$$
\begin{array}{cc}
A_{\text {eff }}=\frac{1-R-T}{1-R} & \text { Eq.5 } \\
S E_{A}=-10 \log \left(1-A_{\text {eff }}\right)=-10 \log \left(\frac{T}{1-R}\right) & \text { Eq.6 } \\
S E_{R}=-10 \log (1-R) & \text { Eq.7 } \\
S E_{T}=S E_{A}+S E_{R}=-10 \log T & \text { Eq.8 }
\end{array}
$$

Thus, from Figure S14, Figure S15, and Eq.1 to Eq.8, the $S E_{T}$ parameter of the electromagnetic shielding sample was shown in Figure S16. 
(a)
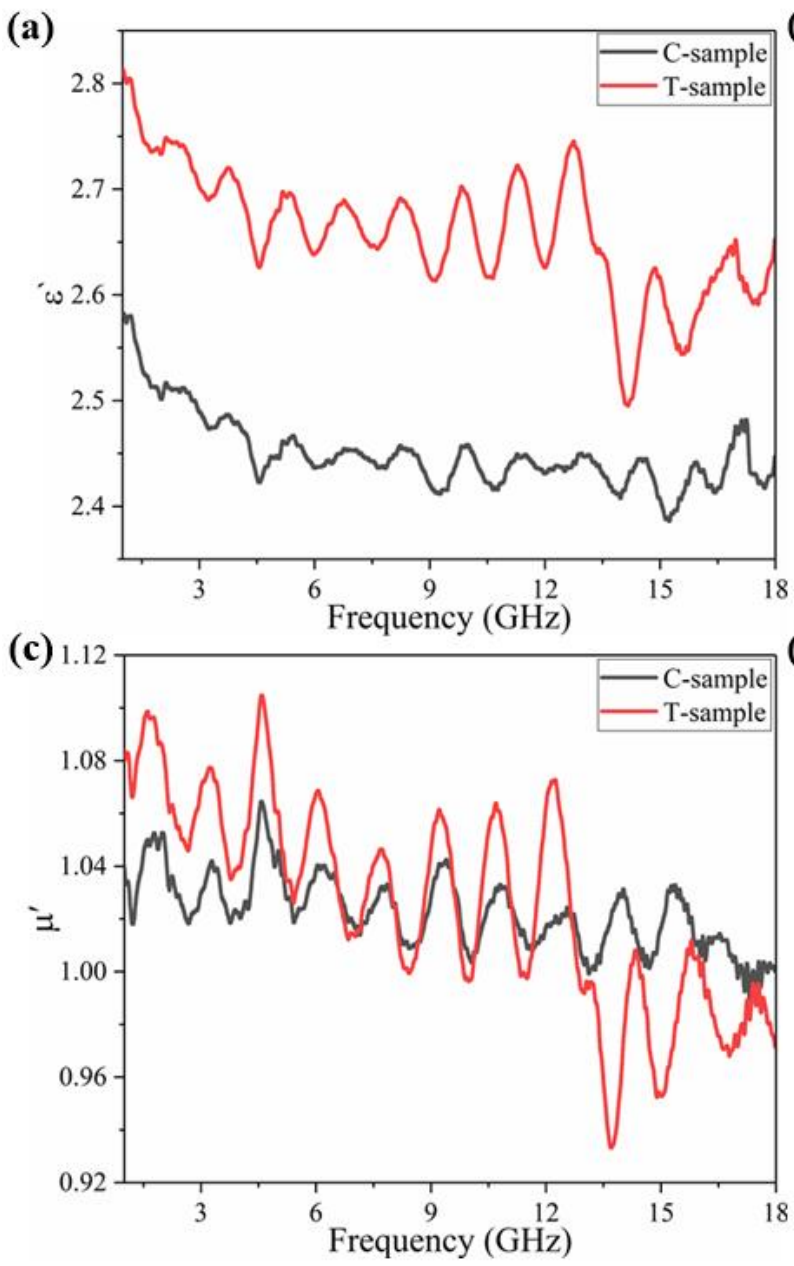

(b)

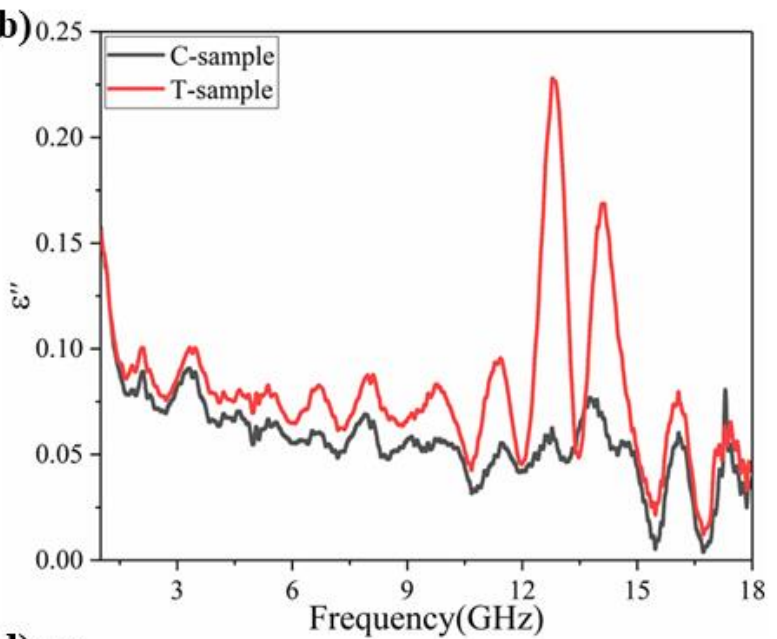

(d)

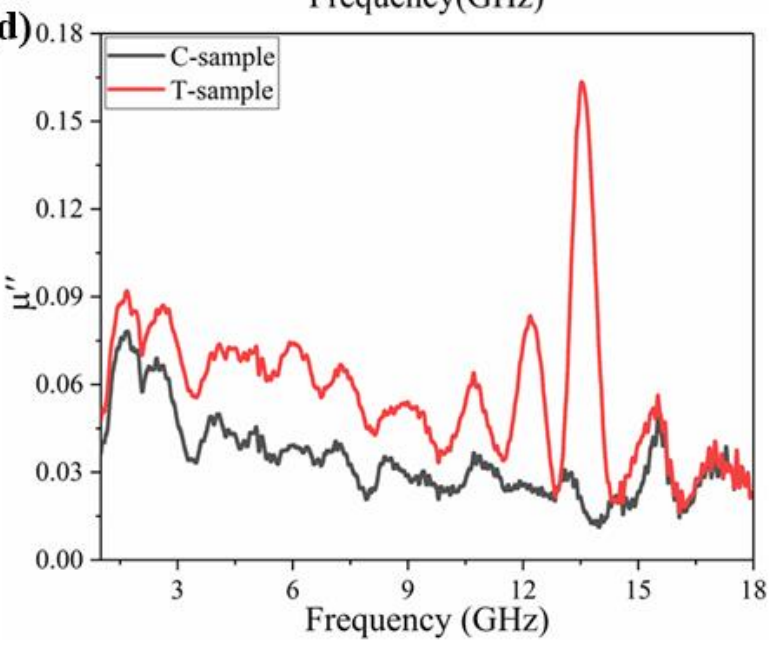

Figure S17. The comparison of the complex permittivity (a) $\varepsilon^{\prime}$ and (b) $\varepsilon^{\prime \prime}$, and complex permeability (c) $\mu^{\prime}$, (d) $\mu^{\prime \prime}$ of electromagnetic wave absorbing sample (EM-DDLC-YS-Ms non-woven fabric for T-sample, and the DDLC-Ms non-woven fabric prepared by the same method as the EM-DDLC-YS-Ms non-woven fabric device but without the magnetic seeds for C-sample).
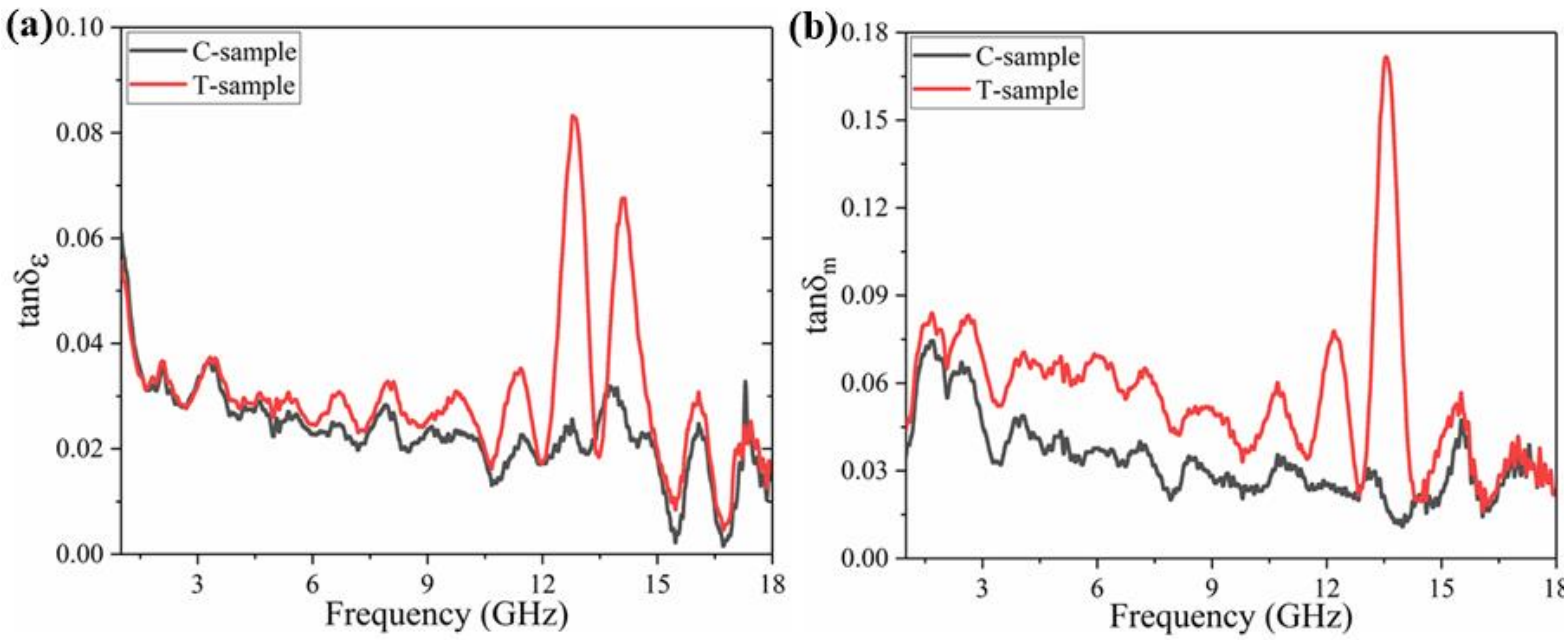

Figure S18. The comparison of the dielectric loss (a) $\tan \delta_{\varepsilon}$ and magnetic loss (b) $\tan \delta_{m}$ of electromagnetic wave absorbing sample (EM-DDLC-YS-Ms non-woven fabric for T-sample, and the DDLC-Ms non-woven fabric prepared by the same method as the EM-DDLC-YS-Ms non-woven fabric device but without the magnetic seeds for C-sample). 

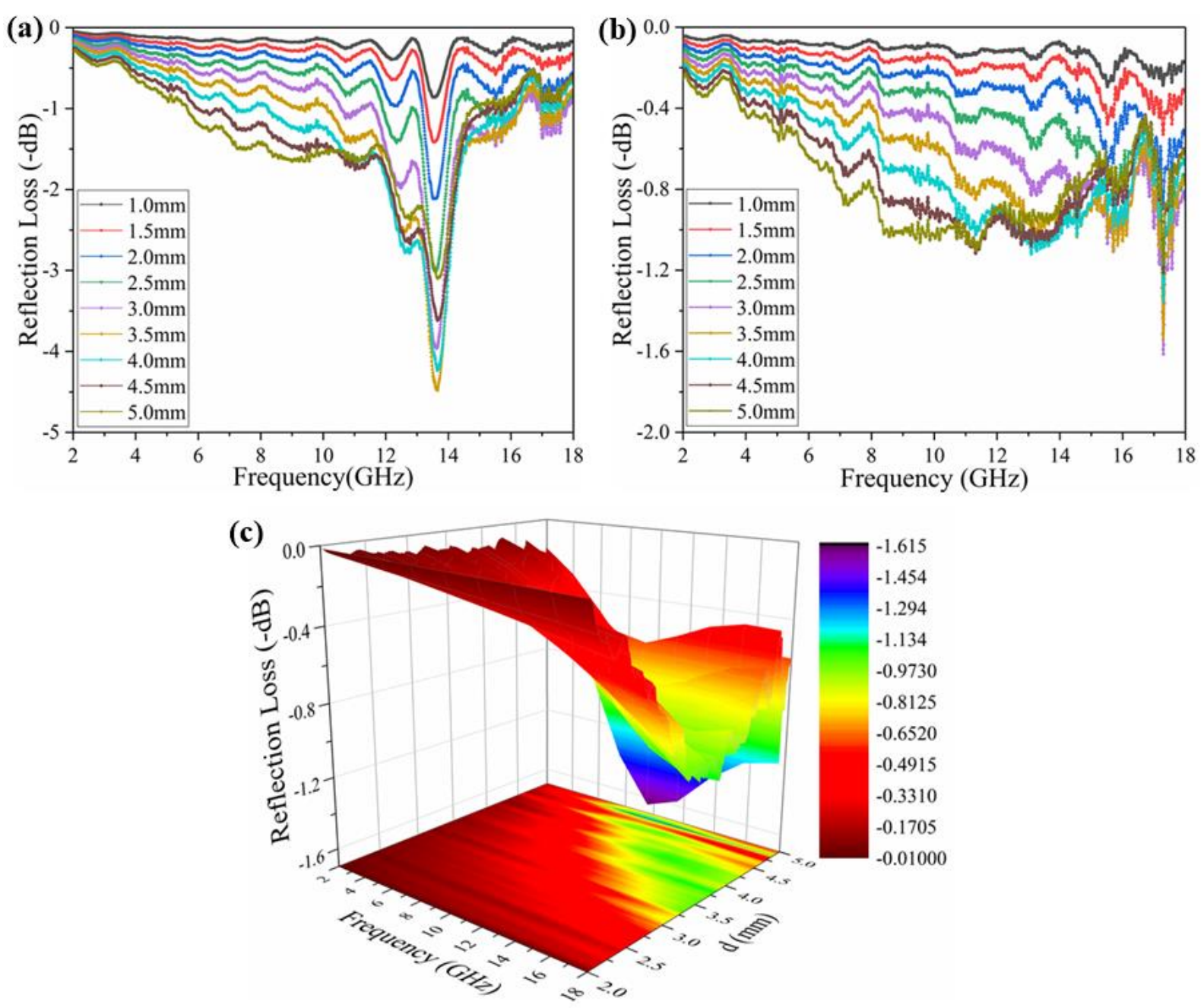

Figure S19. The comparison of the reflection loss curves of electromagnetic wave absorbing samples with different thickness: (a) EM-DDLC-YS-Ms non-woven fabric for T-sample, (b, c) the DDLC-Ms non-woven fabric prepared by the same method as the EM-DDLC-YS-Ms non-woven fabric device but without the magnetic seeds for C-sample. 


\section{Note for Figure S17, Figure S18, and Figure S19:}

In this experiment, the electromagnetic performance parameters of the samples were tested with a microwave network analyzer model PNA-N5244A E5071C (Agilent Co., Ltd, USA). The non-woven fabric fragments (using the microcapsules prepared by the same process with and without magnetic seed particles) and paraffin are mixed evenly according to the mass ratio of 1:2, heated to disperse the paraffin after melting, and pressed into the mold to make a ring sample. The sample size was 7 $\mathrm{mm}$ in outer diameter, $3 \mathrm{~mm}$ in inner diameter, and $2 \mathrm{~mm}$ in thickness, which was marked as test sample (T-sample) and comparative sample (C-sample), respectively. The electromagnetic parameters of the samples were measured by the coaxial method, and the test frequency range was 1-18 GHz. Therefore, the complex permittivity $\varepsilon^{\prime} \varepsilon^{\prime \prime}$ and complex permeability $\mu^{\prime} \mu^{\prime \prime}$ parameters of the sample were shown in Figure S17.

The dielectric loss $\tan \delta_{\varepsilon}$ and magnetic loss $\tan \delta_{m}$ represents the electromagnetic loss of the material, and the greater the parameters, the stronger the attenuation of the electromagnetic wave by the material. Using the experimentally measured complex permittivity and complex permeability, the materials $\tan \delta_{\varepsilon}$ and $\tan \delta_{m}$ can be calculated as shown in Figure S18. The calculation formula is as follows:

$$
\begin{gathered}
\tan \delta_{\varepsilon}=\frac{\varepsilon^{\prime \prime}}{\varepsilon^{\prime}} \\
\tan \delta_{m}=\frac{\mu^{\prime \prime}}{\mu^{\prime}}
\end{gathered}
$$

The electromagnetic wave absorption intensity of the sample can be expressed by the reflection loss $\mathrm{R}_{\mathrm{L}}(\mathrm{dB})$. Using the complex dielectric constant and complex permeability measured by the experiment, the reflection loss of the material can be calculated as shown in Figure S19 and Figure 5c. The calculation formula is as follows: 


$$
R_{L}(d B)=-20 \lg \left|\frac{\left(Z_{i n}-1\right)}{\left(Z_{i n}+1\right)}\right|
$$

Where $Z_{\text {in }}$ is the normalized input impedance of the absorber, which calculation formula is as follows:

$$
Z_{\text {in }}=\sqrt{\frac{\mu_{r}}{\varepsilon_{r}}} \tanh \left(-j \frac{2 \pi f d}{c} \sqrt{\mu_{r} \varepsilon_{r}}\right)
$$

In the formula, $\varepsilon_{r}$ and $\mu_{r}$ are the complex permittivity and complex permeability of the absorber sample, respectively. $c, f$, and $d$ were the propagation speed of the electromagnetic wave in free space $\left(3 * 10^{8} \mathrm{~m} / \mathrm{s}\right)$, frequency $(\mathrm{Hz})$, and the thickness of the absorber sample $(\mathrm{mm})$. When $\mathrm{R}_{\mathrm{L}}=-10 \mathrm{~dB}$ means that $90 \%$ of the waves are absorbed by the absorber, thus in practical applications, $R_{L}=-10 \mathrm{~dB}$ is often used as the measurement point of the material's absorbing performance. The frequency band of $\mathrm{R}_{\mathrm{L}}<-10 \mathrm{~dB}$ represents the effective absorbing frequency band of the absorber. 


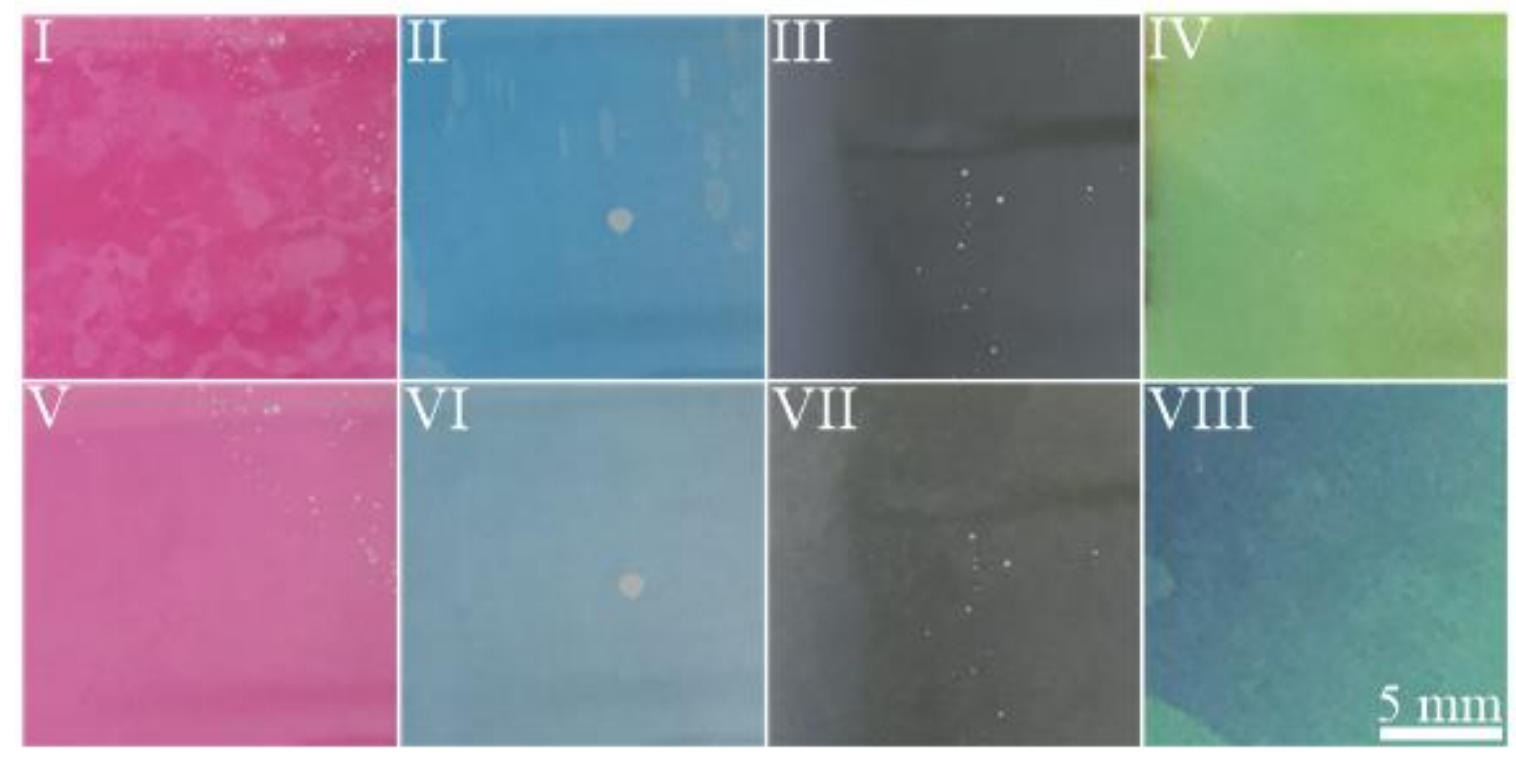

Figure S20. Photographs of EMs-Fabric prepared by other different DDLC and CLC systems under applied (I, II, III, IV) and removed (V, VI, VII, VIII) electric field state. 


\section{Supplementary Movies}

Movie S1. The color change of the EM-DDLC-YS-Ms during applying and removing voltage. 


\section{Supplementary Description}

According to previous literature (J. Appl. Phys. 2008, 103, 084505 DOI: 10.1063/1.2903063; Opt. Spectrosc. 2003, 94, 595-599 DOI: 10.1134/1.1570487), DDLCs are more sensitive to the confined geometry and the polymer matrix in PDLC, and which sphericity and size of polymer cavity are difficult to control and hinders the precise study of photoelectric performance in micro-scale. Besides, it is also difficult to apply to the flexible wearable display field due to its membrane structure. Compared with PDLC, the uniform-sized monodispersed DDLC microcapsules can be obtained via the adoption of the encapsulation method, and which can provide enhanced light transmission by preventing the light scattering caused by differently sized DDLC droplets. Moreover, DDLC encapsulated into the polymer shell would not only form a three-dimensional independent structure but also protect DDLC from the external environment and thus expands its application domain and prolongs the service life.

In particular, as the reviewer suggested, the creative experience of two works whom may be interested in is briefly explained below:

The idea of the "June 2020 study" 5 came out in November 2017, and the experiment work was mainly completed in September 2019. Inspired by the original superimposed three-color multi-twisted dye-doped liquid crystal display, we are trying to microencapsulate this display mode so that it can be applied to flexible multi-material electrochromic films and wearable smart textiles. Thus, we designed a yolk-shell microcapsules structure containing two separate DDLC layers of different colors are designed to produce multicolored DDLC microcapsules. Although the fabrication of various yolk-shell materials has received significant attention in recent decades, the construction of yolk-shell structures is mainly achieved through templating, layer-by-layer method. These methods typically require tedious and costly synthesis procedures, as well as unavoidable 
contamination of the liquid crystals. Also, previous studies were conducted on the nanoscale, which is disadvantageous for determining the photoelectric properties of DDLC microcapsules. Thus, the synthesis of yolk-shell DDLC microcapsules through mild and non-sacrificial scaffolding routes or methods remains a challenge. After a long exploration, we find an interesting phenomenon that non-ionic surfactant Brij-35 can change the surface hydrophilicity of the particle. Subsequently, we introduce the Brij-35 as "oil-water channel switching", and report a novel, facile, and mild surfactant-inspired engineering strategy to synthesize uniform yolk-shell DDLC microcapsules based on seed emulsion polymerization. The microcapsules represent a novel synthesis method inspired by the surface property switching abilities of leaves, which could be applied for the fabrication of other structured microdevices.

Back to this work, the idea of it came out in June 2018, and the experiment work was mainly completed in May 2020. To broaden the spectral modulation capability and functional characteristics of smart liquid crystal microcapsules, magnetic carriers as promising materials because of the combination of their unique magnetic features and microwave absorbing capacity were introduced into the microcapsule system. However, the magnetic particles were difficult to form a single individual microcapsule contain with DDLC due to the poor compatibility with DDLC and self-aggregation characteristics. Initially, we tried to use the template method to construct this yolk-shell structure (a reference to the Zhao et' work J. Am. Chem. Soc. 2017, 139, 15486-15493, DOI: $10.1021 /$ jacs.7b09055.). However, it is difficult to obtain micron-level microcapsules with uniform particle size by template method, and the residue of the reagents will easily cause the contamination of dye-doped liquid crystals. Meanwhile, the "oil-water channel switching" characteristic of Brij-35 was found and investigated. Thus, we adopted a similar strategy to ensure DDLC/MMA adsorption on the magnetic seeds surface completely and keep good dispersion in the 
water to avoid agglomeration behavior in the synthesis process. Also, we quote this reference ("June 2020 study") in the manuscript and simplify the sections of synthesis and characterization to avoid cumbersome mechanism discussions. 


\section{Supplementary References}

(1) Grigoriadis, C.; Duran, H.; Steinhart, M.; Kappl, M.; Butt, H.-J. r.; Floudas, G. Suppression of Phase Transitions in a Confined Rodlike Liquid Crystal. ACS Nano 2011, 5 (11), 9208-9215.

(2) Miyano, K. Wall-Induced Pretransitional Birefringence: a New Tool to Study Boundary Aligning Forces in Liquid Crystals. Physical Review Letters 1979, 43 (1), 51.

(3) Peng, H.; Jiang, W.; Liu, Q.; Chen, G.; Ni, M.; Liang, F.; Liao, Y.; Xie, X.; Smalyukh, I. I. Liquid Crystals under Confinement in Submicrometer Capsules. Langmuir 2018, 34 (37), 10955-10963.

(4) Yan, D. X.; Pang, H.; Li, B.; Vajtai, R.; Xu, L.; Ren, P. G.; Wang, J. H.; Li, Z. M. Structured Reduced Graphene Oxide/Polymer Composites for Ultra-Efficient Electromagnetic Interference Shielding. Advanced Functional Materials 2015, 25 (4), 559-566.

(5) Sheng, M.; Zhang, L.; West, J. L.; Fu, S. Multicolor Electrochromic Dye-Doped Liquid Crystal Yolk-Shell Microcapsules. ACS Applied Materials \& Interfaces 2020, 12 (26), 29728-29736. 